\title{
Spherical transverse M5-branes from the plane wave matrix model
}

\author{
Yuhma Asano, $^{a}$ Goro Ishiki, ${ }^{b, c}$ Shinji Shimasaki ${ }^{d}$ and Seiji Terashima ${ }^{e}$ \\ ${ }^{a}$ School of Theoretical Physics, Dublin Institute for Advanced Studies, \\ 10 Burlington Road, Dublin 4, Ireland \\ ${ }^{b}$ Tomonaga Center for the History of the Universe, University of Tsukuba, \\ Tsukuba, Ibaraki 305-8571, Japan \\ ${ }^{c}$ Graduate School of Pure and Applied Sciences, University of Tsukuba, \\ Tsukuba, Ibaraki 305-8571, Japan \\ ${ }^{d}$ Research and Education Center for Natural Sciences, Keio University, \\ Hiyoshi 4-1-1, Yokohama, Kanagawa 223-8521, Japan \\ ${ }^{e}$ Yukawa Institute for Theoretical Physics, Kyoto University, \\ Kyoto 606-8502, Japan \\ E-mail: yuhma@stp.dias.ie, ishiki@het.ph.tsukuba.ac.jp, \\ shinji.shimasaki@keio.jp, terasima@yukawa.kyoto-u.ac.jp
}

ABSTRACT: We consider matrix theoretical description of transverse M5-branes in Mtheory on the 11-dimensional maximally supersymmetric pp-wave background. We apply the localization to the plane wave matrix model (PWMM) and show that the transverse spherical fivebranes with zero light cone energy in M-theory are realized as the distribution of low energy moduli of the $\mathrm{SO}(6)$ scalar fields in PWMM.

Keywords: Field Theories in Lower Dimensions, M(atrix) Theories, Supersymmetric Gauge Theory, M-Theory

ArXiv EPrint: 1711.07681 


\section{Contents}

1 Introduction 1

2 M-theory on the pp-wave background 5

2.1 Spherical M2-brane 5

$\begin{array}{lll}2.2 & \text { Spherical M5-brane } & 7\end{array}$

2.3 Decoupling limits 8

$\begin{array}{lll}3 & \text { The plane wave matrix model } & 9\end{array}$

4 Spherical M5-branes from PWMM 11

4.1 Localization in PWMM 11

$\begin{array}{lll}4.2 & \text { Coincident M5-branes from the simplest partition } & 12\end{array}$

$\begin{array}{lll}4.3 & \text { Multiple M5-branes from generic partitions } & 16\end{array}$

5 Spherical M2-branes from PWMM 18

$\begin{array}{llr}6 & \text { Summary and discussion } & 19\end{array}$

$\begin{array}{ll}\text { A Localization in PWMM } & \mathbf{2 0}\end{array}$

$\begin{array}{ll}\text { A.1 Off-shell supersymmetry of PWMM } & 20\end{array}$

A.2 Saddle point of the localization 21

$\begin{array}{lll}\text { A.3 Ghost fields } & 22\end{array}$

A.4 1-loop determinants 24

A.5 Derivation of 1-loop determinants 26

$\begin{array}{ll}\text { B The saddle point equation } & 31\end{array}$

C Solving the saddle point equation $\quad 32$

C.1 For the simplest partition $\quad 32$

C.2 For the generic partition 33

$\begin{array}{ll}\text { D Eigenvalue distribution in the M2-brane limit } & 35\end{array}$

\section{Introduction}

Matrix models are conjectured to give nonperturbative formulations of M-theory [1]. This formulation is expected to realize a second quantization of M-theory, which contains all the fundamental objects in the theory. However, the description of states with M5-branes in the matrix models has not been established yet. Understanding this problem will shed light on the matrix-model formulation of M-theory. 
In this paper, we focus on M-theory defined on the maximally supersymmetric ppwave solution of the 11-dimensional supergravity and consider the description of certain M5-branes living in this geometry in terms of the matrix model. On this background, there exist stable spherical M2- and M5- branes with zero light cone energy. According to the matrix-model conjecture, objects with zero light cone energy should be realized as vacuum states in the corresponding matrix model. Hence, these spherical branes should also be realized as certain vacuum states in the matrix model. In this paper, we investigate this relation in detail by using the localization method.

The matrix model for M-theory on the pp-wave background is called the plane wave matrix model (PWMM) [2]. This model is given by a mass deformation of the BFSS matrix model [1], where the mass parameter is proportional to the three form flux on the pp-wave geometry. Because of the mass deformation, PWMM possesses many discretely degenerate vacua, unlike the BFSS matrix model. The relation between these vacua and objects with vanishing light cone energy in M-theory was proposed in $[2,3]$. Here, in particular, the vacua corresponding to the above mentioned spherical M5-brane and its multiple generalization were also specified. For the case of a single M5-brane, this correspondence was tested by comparing the BPS protected mass spectra of PWMM with that of the M5-brane [3].

Let us review this proposal in more detail. The vacua of PWMM, which preserve all the supersymmetry, are given by the fuzzy sphere [4] and are labeled by $N$-dimensional representations of the $\mathrm{SU}(2)$ Lie algebra, where $N$ is the matrix size of PWMM. Generally, the classical vacuum configuration in PWMM takes the form of

$$
X_{i} \propto L_{i}, \quad(i=1,2,3)
$$

where $X_{i}$ are the $\mathrm{SO}(3)$ scalar fields in PWMM and the other fields are vanishing at the vacuum. $L_{i}$ are $N$-dimensional representation matrices of the $\mathrm{SU}(2)$ generators. Any $N$-dimensional representation gives a supersymmetric vacuum and, in general, the representation is reducible. Then, one can make an irreducible decomposition:

$$
L_{i}=\bigoplus_{s=1}^{\Lambda} L_{i}^{\left[n_{s}\right]} \otimes 1_{N_{2}^{(s)}} .
$$

Here, $L_{i}^{\left[n_{s}\right]}$ are the generators in the $n_{s}$-dimensional irreducible representation and $N_{2}^{(s)}$ represents the multiplicity of the $s$ th representation. Hence, the vacua can be labeled by a set of integers $\left\{\Lambda, N_{2}^{(s)}, n_{s} \mid s=1,2, \cdots, \Lambda\right\}$ satisfying $\sum_{s=1}^{\Lambda} n_{s} N_{2}^{(s)}=N$.

From this structure of the vacua, we can immediately find the structure of the spherical M2-brane in M-theory. The fuzzy sphere is a regularization of a smooth two-dimensional sphere. In the commutative limit, where $N_{2}^{(s)}$ are fixed while $n_{s}$ go to infinity, smooth two-spheres are realized from the fuzzy sphere. One can naturally expect that this smooth sphere is the spherical M2-brane with zero light cone energy.

On the other hand, in [2], the spherical M5-brane was conjectured to be realized as the trivial vacuum of PWMM, where all the fields are vanishing. This is the case where the representation in (1.1) is a direct sum of $N$ trivial representations. Furthermore, the conjecture was generalized to the case of multiple spherical M5-branes [3]. In these 

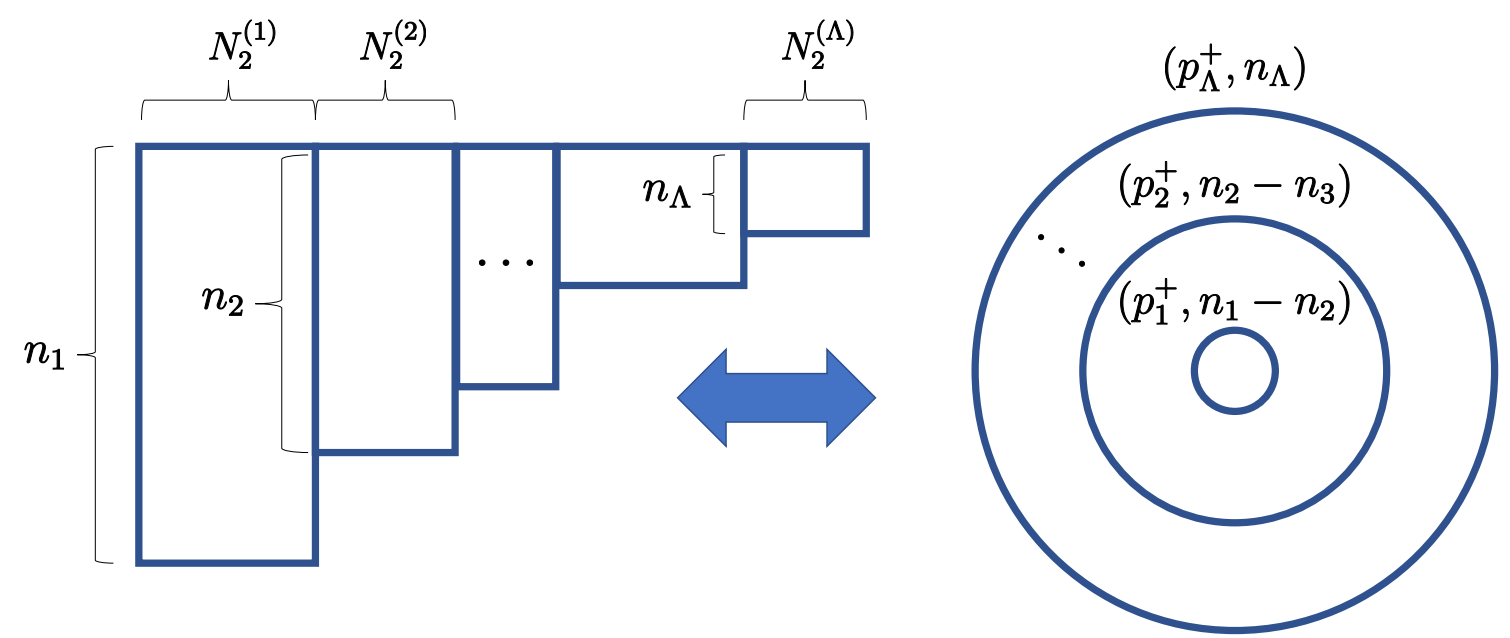

Figure 1. Correspondence between partitions and configurations of M5-branes.

conjectures, the M5-branes are considered to be realized in the limit such that $n_{s}$ are fixed and $N_{2}^{(s)}$ go to infinity in (1.2).

In order to describe this limit more precisely, let us introduce Young diagrams associated with the partition of (1.2). In the decomposition (1.2), we assume that $n_{1}>n_{2}>$ $\cdots>n_{\Lambda}$ without loss of generality. Then we consider a Young diagram which consists of $N_{2}^{(1)}$ columns with length $n_{1}, N_{2}^{(2)}$ columns with length $n_{2}$, and so on. See figure 1 . The conjecture states that when the lengths of some rows go to infinity, such rows correspond to the spherical M5-branes, where the light cone momentum of each M5-brane is proportional to the length of each row. For example, in figure 1, let us consider the limit where all $N_{2}^{(s)}$ go to infinity with the same order while all $n_{s}$ are fixed. This limit corresponds to a situation in M-theory such that there are $\Lambda$ stacks of spherical M5-branes, where the $s$ th stack is made of $n_{s}-n_{s+1}$ M5-branes ${ }^{1}$ with light cone momentum

$$
p_{s}^{+}=\sum_{r=1}^{s} N_{2}^{(r)} / R,
$$

where $R$ is the radius of the light like circle. ${ }^{2}$ Note that the total light cone momentum is given by $p^{+}=\sum_{s=1}^{\Lambda}\left(n_{s}-n_{s+1}\right) p_{s}^{+}$and this is equal to $N / R$. Note also that $N_{5}:=$ $\max \left\{n_{s} \mid s=1,2, \ldots\right\}=n_{1}$ corresponds to the total number of M5-branes.

This conjecture is highly nontrivial. For example, let us consider the simplest partition with $\Lambda=1, n_{1}=1, N_{2}^{(1)}=N$, which corresponds to the trivial vacuum of PWMM. At the classical level, the vacuum configuration is just vanishing, so that we can not see any structure of the M5-brane. For example, it looks seemingly impossible to reproduce geometric information of the spherical M5-brane (the radius etc.) from the trivial configu-

\footnotetext{
${ }^{1}$ For $s=\Lambda$, we define $n_{\Lambda+1}:=0$.

${ }^{2}$ As we will see in the next section, the radius of a single (i.e. not coincident) M5-brane is proportional to $\left(p^{+}\right)^{1 / 4}$. Thus, larger $p^{+}$gives a larger radius. Though this relation had never been derived for coincident M5-branes, our results discussed below shows that this is also true for coincident M5-branes. Figure 1 is based on this picture, so that the $s$ th stack has a larger radius than $(s-1)$ th stack.
} 
ration. Nevertheless, the conjecture claims that a single spherical M5-brane is realized in the trivial vacuum.

To bridge this gap, one needs to recall that M-theory is conjectured to be realized in an appropriate large- $N$ limit of PWMM, where the coupling constant also becomes very large as the matrix size $N$ goes to infinity. Thus, one has to deal with the strongly coupled regime of PWMM, in order to understand the description of M5-branes. In the strong coupling region, there must be a large quantum fluctuation around the classical vacuum configuration. Thus, typical configurations of matrices will be very different from the classical configuration. There is a possibility that the spherical M5-branes are formed as a typical configuration of matrices in the strong coupling region of PWMM. ${ }^{3}$

In this paper, we investigate this possibility by directly studying the strong coupling regime of PWMM. The limit we consider is

$$
N_{2}^{(s)} \rightarrow \infty, \quad n_{s} \text { fixed, } N_{2}^{(s)} / N_{2}^{(t)} \text { fixed }
$$

for any $s, t=1,2, \cdots, \Lambda$. This limit corresponds to $\Lambda$ stacks of M5-branes with different radii as shown in figure 1. In addition, we also scale the coupling constant of PWMM in such a way that the M5-branes decouple with the bulk gravity and only the degrees of freedom on the M5-branes become relevant [3]. This decoupling limit turns out to be the strong coupling limit in the 't Hooft limit of PWMM, as we will describe in the next section. In this decoupling limit, we apply the localization to PWMM and reduce some BPS correlation functions to certain eigenvalue integrals. By evaluating the eigenvalue integral, we argue that the eigenvalue distribution of the low energy modes of the $\mathrm{SO}(6)$ scalar fields forms $\Lambda$ stacks of spherical shells and coincides with the expected configuration of the spherical M5-branes in M-theory. ${ }^{4}$ In particular, we show that, for a single M5-brane, the radius of the shell completely agrees with the value computed by using the classical Dirac-Nambu-Goto action of a single M5-brane. This result strongly supports the proposal of [3] and shows that PWMM indeed contains the multiple M5-brane states. We also apply the same argument to M2-branes and show that the spherical M2-brane can be described in a similar way using the eigenvalue integral.

This paper is organized as follows. In section 2, we review M-theory on the pp-wave background. We show that there exist spherical M2- and M5- branes with zero light cone energy on this background. We also compute the radii of these objects. In section 3, we review PWMM. In section 4, we apply the localization to PWMM and evaluate the moduli distribution of scalar fields. We show that the distribution agrees with the configuration of the spherical M5-branes. In section 5, we consider the case of M2-branes. In section 6, we summarize our results and discuss the low energy theory of PWMM.

\footnotetext{
${ }^{3}$ See also [5] for the description of M5-branes in a different matrix model.

${ }^{4} \mathrm{~A}$ part of this result was briefly reported in the letter [6] for the case of concentric M5-branes. In this paper, we not only describe the technical details of [6] but also generalize the result of [6] to the most general configurations of the spherical M5-branes.
} 


\section{M-theory on the pp-wave background}

In this section, we review M-theory on the maximally supersymmetric plane wave background in the 11-dimensional supergravity. The background geometry is given by

$$
\begin{aligned}
d s^{2} & =g_{\mu \nu} d x^{\mu} d x^{\nu}=-2 d x^{+} d x^{-}+\sum_{A=1}^{9} d x^{A} d x^{A}-\left(\frac{\mu^{2}}{9} \sum_{i=1}^{3} x^{i} x^{i}+\frac{\mu^{2}}{36} \sum_{a=4}^{9} x^{a} x^{a}\right) d x^{+} d x^{+}, \\
F_{123+} & =\mu,
\end{aligned}
$$

where $\mu$ is the flux parameter of the three form field. ${ }^{5}$ We will see that spherical M2-brane and M5-brane exist as the lowest energy states with respect to the light cone Hamiltonian. We refer the method in [7] for the calculation in this section.

\subsection{Spherical M2-brane}

We first consider a single M2-brane in the background (2.1). The bosonic part of the M2-brane action is given by the Dirac-Nambu-Goto action plus a Chern-Simons term as

$$
S_{\mathrm{M} 2}=-T_{\mathrm{M} 2} \int d^{3} \sigma \sqrt{-\operatorname{det} h_{\alpha \beta}}+T_{\mathrm{M} 2} \int C_{3} .
$$

Here, $h_{\alpha \beta}$ is the induced metric,

$$
h_{\alpha \beta}=g_{\mu \nu}(X) \partial_{\alpha} X^{\mu} \partial_{\beta} X^{\nu},
$$

for the embedding function $X^{\mu}(\sigma)$. The overall constant $T_{\mathrm{M} 2}$ in $(2.2)$ is the tension of M2-brane given by

$$
T_{\mathrm{M} 2}=\frac{1}{(2 \pi)^{2} l_{p}^{3}},
$$

where $l_{p}$ stands for the Planck length. By introducing a symmetric auxiliary field $\gamma_{\alpha \beta}$, we rewrite the action into the Polyakov type:

$$
S_{\mathrm{M} 2}=-\frac{T_{M 2}}{2} \int d^{3} \sigma \sqrt{-\gamma}\left(\gamma^{\alpha \beta} g_{\mu \nu}(X) \partial_{\alpha} X^{\mu} \partial_{\beta} X^{\nu}-1\right)+T_{\mathrm{M} 2} \int C_{3} .
$$

This action has a diffeomorphism symmetry for the worldvolume coordinates $\sigma^{\alpha}=$ $\left(\sigma^{0}, \sigma^{1}, \sigma^{2}\right)$ of the membrane. If we consider an M2-brane with topology $R \times \Sigma$, where $R$ is the time direction and $\Sigma$ is a Riemann surface, we can fix this symmetry by putting

$$
\gamma_{0 a}=0, \quad \gamma_{00}=-\frac{4}{\nu^{2}} \operatorname{det} h_{a b},
$$

where $a, b=1,2$ and the determinant is taken in this $2 \times 2$ subspace. $\nu$ is a constant which will be related to the light cone momentum of the M2-brane below. Then, the action becomes

$$
\begin{aligned}
S_{\mathrm{M} 2} & =\frac{T_{\mathrm{M} 2} \nu}{4} \int d^{3} \sigma\left(h_{00}-\frac{4}{\nu^{2}} \operatorname{det} h_{a b}\right)+T_{\mathrm{M} 2} \int C_{3} \\
& =\frac{T_{\mathrm{M} 2} \nu}{4} \int d^{3} \sigma\left(-2 \dot{X}^{-}+\left(\dot{X}^{A}\right)^{2}-\frac{\mu^{2}}{9}\left(X^{i}\right)^{2}-\frac{\mu^{2}}{36}\left(X^{a}\right)^{2}-\frac{2}{\nu^{2}}\left\{X^{A}, X^{B}\right\}^{2}\right)+T_{\mathrm{M} 2} \int C_{3} .
\end{aligned}
$$

\footnotetext{
${ }^{5}$ Throughout this paper, we mainly use the notation that $\mu, \nu=0,1,2, \cdots, 10, A, B=1,2, \cdots, 9$, $i, j=1,2,3$ and $a, b=4,5, \cdots, 9$.
} 
Here, in the second line, we have introduced a canonical Poisson bracket on the membrane defined by $\{f, g\}=\epsilon^{a b}\left(\partial_{a} f\right)\left(\partial_{b} g\right)$ for each fixed $\sigma^{0}$. In terms of the Poisson bracket, the Chern-Simons term can be written as

$$
\int C_{3}=\frac{\mu}{6} \int d^{3} \sigma \epsilon_{i j k} X^{i}\left\{X^{j}, X^{k}\right\} .
$$

The gauge fixing condition (2.6) as well as the equation of motion of the auxiliary field produce the following constraints:

$$
\begin{aligned}
g_{\mu \nu} \dot{X}^{\mu} \dot{X}^{\nu} & =-\frac{2}{\nu^{2}} g_{\mu \nu} g_{\rho \sigma}\left\{X^{\mu}, X^{\rho}\right\}\left\{X^{\nu}, X^{\sigma}\right\} \\
g_{\mu \nu} \dot{X^{\mu}} \partial_{a} X^{\nu} & =0 .
\end{aligned}
$$

From the second constraint, it also follows that

$$
\left\{g_{\mu \nu} \dot{X}^{\mu}, X^{\nu}\right\}=0 .
$$

Thus, the system is reduced to the theory (2.7) with these constraints imposed.

The constraints (2.9) can be explicitly solved in the light cone gauge,

$$
X^{+}(\sigma)=\sigma^{0} .
$$

Here, we have defined $X^{ \pm}$by

$$
X^{ \pm}=\frac{1}{\sqrt{2}}\left(X^{0} \pm X^{10}\right) .
$$

We then consider the Hamilton formalism. We denote by $P^{\mu}$ the canonical conjugate momentum of $X^{\mu}$. The total light cone momentum is then given by

$$
p^{+}=\int d^{2} \sigma P^{+}=2 \pi \nu T_{\mathrm{M} 2},
$$

where we have chosen the spacial coordinates such that they have a volume $\int d^{2} \sigma=4 \pi$. This relates the constant $\nu$ to the light cone momentum. The Hamiltonian is given by

$$
\begin{aligned}
H_{\mathrm{M} 2}=\int d^{2} \sigma & {\left[\frac{V_{2}}{2 p^{+}}\left(P_{A}^{2}+\frac{T_{\mathrm{M} 2}^{2}}{2}\left\{X^{A}, X^{B}\right\}^{2}\right)\right.} \\
& \left.+\frac{p^{+}}{2 V_{2}}\left(\frac{\mu^{2}}{9}\left(X^{i}\right)^{2}+\frac{\mu^{2}}{36}\left(X^{a}\right)^{2}\right)-\frac{\mu T_{\mathrm{M} 2}}{6} \epsilon_{i j k} X^{i}\left\{X^{j}, X^{k}\right\}\right],
\end{aligned}
$$

where, $V_{2}$ is the volume of the unit sphere, $V_{2}=4 \pi$. The remaining constraint $(2.10)$ is written in terms of the transverse components $X^{A}$ as

$$
\left\{P^{A}, X_{A}\right\}=0
$$

Now, let us consider a vacuum configuration, which minimizes the Hamiltonian (2.14). Note that the potential for $X^{i}$ forms a perfect square,

$$
\frac{p^{+} \mu^{2}}{18 V_{2}}\left(X_{i}-\frac{3 V_{2} T_{\mathrm{M} 2}}{2 \mu p^{+}} \epsilon_{i j k}\left\{X^{i}, X^{j}\right\}\right)^{2} .
$$


From this, we find that the vacuum configuration is given by

$$
X^{i}=r_{\mathrm{M} 2} x^{i}, \quad X^{a}=0, \quad P_{A}=0,
$$

where $x^{i}$ are the embedding function of the unit sphere in $R^{3}$ satisfying

$$
x^{i} x^{i}=1, \quad\left\{x^{i}, x^{j}\right\}=\epsilon^{i j k} x_{k} .
$$

The radius is also determined as

$$
r_{\mathrm{M} 2}=\frac{\mu p^{+}}{12 \pi T_{\mathrm{M} 2}} .
$$

The configuration (2.17) obviously has the spherical shape. Thus we see that, in M-theory on the pp-wave background, there exists a spherical zero energy M2-brane with the radius given by (2.19).

\subsection{Spherical M5-brane}

Then, let us consider a single M5-brane. We start from the bosonic part of the action,

$$
S_{\mathrm{M} 5}=-T_{\mathrm{M} 5} \int d^{6} \sigma \sqrt{-\operatorname{det} h_{\alpha \beta}}+T_{\mathrm{M} 5} \int C_{6},
$$

where $d C_{6}=* F_{4}$ and the tension is written as

$$
T_{\mathrm{M} 5}=\frac{1}{(2 \pi)^{5} l_{p}^{6}} .
$$

We can apply the computation in the previous subsection to (2.20). Then, we can obtain the light-cone Hamiltonian for the M5-brane,

$$
\begin{aligned}
H_{\mathrm{M} 5}=\int d^{5} \sigma & {\left[\frac{V_{5}}{2 p^{+}}\left(P_{A}^{2}+\frac{T_{\mathrm{M} 5}^{2}}{5 !}\left\{X^{A_{1}}, \cdots, X^{A_{5}}\right\}^{2}\right)\right.} \\
& \left.+\frac{p^{+}}{2 V_{5}}\left(\frac{\mu^{2}}{9}\left(X^{i}\right)^{2}+\frac{\mu^{2}}{36}\left(X^{a}\right)^{2}\right)-\frac{\mu T_{\mathrm{M} 5}}{6 !} \epsilon_{a_{1} a_{2} \cdots a_{6}} X^{a_{1}}\left\{X^{a_{2}}, \cdots, X^{a_{6}}\right\}\right] .
\end{aligned}
$$

Here, $V_{5}$ is the volume of the unit 5-dimensional sphere, $V_{5}=\pi^{3}$. The curly bracket with five entries in (2.22) is the 5-dimensional analogue of the Poisson bracket defined by

$$
\left\{f_{1}, \cdots, f_{5}\right\}=\epsilon^{a_{1} \cdots a_{5}}\left(\partial_{a_{1}} f_{1}\right) \cdots\left(\partial_{a_{5}} f_{5}\right) .
$$

We notice that the potential terms of $X^{a}$ forms a perfect square,

$$
\frac{p^{+} \mu^{2}}{72 V_{5}}\left(X_{a_{1}}-\frac{6 V_{5} T_{\mathrm{M} 5}}{5 ! \mu p^{+}} \epsilon_{a_{1} a_{2} \cdots a_{6}}\left\{X^{a_{2}}, \cdots, X^{a_{6}}\right\}\right)^{2} .
$$

Thus, we find that the vacuum configuration is given by a spherical fivebrane of the form,

$$
X^{i}=0, \quad X^{a}=r_{\mathrm{M} 5} x^{a}, \quad P_{A}=0,
$$

where $x^{a}$ are the embedding function of the unit 5 -sphere into $R^{6}$ satisfying

$$
x^{a} x^{a}=1, \quad\left\{x^{a_{1}}, \cdots, x^{a_{5}}\right\}=\epsilon^{a_{1} a_{2} \cdots a_{6}} x_{a_{6}} .
$$

The radius of the fivebrane is determined as

$$
r_{\mathrm{M} 5}=\left(\frac{\mu p^{+}}{6 \pi^{3} T_{\mathrm{M} 5}}\right)^{1 / 4} .
$$




\subsection{Decoupling limits}

In this paper, we focus on the limits in which the radii of the spherical M2- and M5- branes become very large and only the degrees of freedom on these branes survive for low energy physics [3].

Let us introduce the radius $r=\sqrt{x^{i} x^{i}}$ of the two sphere on which the M2-brane is wrapping. The metric (2.1) is written as

$$
\begin{aligned}
d s^{2} & =-2 d x^{+} d x^{-}-\frac{\mu^{2} r^{2}}{9} d x^{+} d x^{+}+r^{2} d \Omega_{2}^{2}+\cdots \\
& =-\frac{\mu^{2} r^{2}}{9} d \tilde{x}^{+} d \tilde{x}^{+}+\frac{9}{\mu^{2} r^{2}} d \tilde{x}^{-} d \tilde{x}^{-}+r^{2} d \Omega_{2}^{2}+\cdots,
\end{aligned}
$$

where $\cdots$ represents the other terms which are irrelevant in this discussion and we have defined $\tilde{x}^{ \pm}$by $\tilde{x}^{+}=x^{+}+\frac{9}{\mu^{2} r^{2}} x^{-}, \tilde{x}^{-}=x^{-}$. Note that $\tilde{x}^{ \pm}$have the periodicity

$$
\left(\tilde{x}^{+}, \tilde{x}^{-}\right) \sim\left(\tilde{x}^{+}, \tilde{x}^{-}\right)+\left(9 R /(\mu r)^{2}, R\right),
$$

where $R$ is the radius of the original compactified circle along the light-like direction. Since the shift of $\tilde{x}^{+}$is much smaller than that of $\tilde{x}^{-}$in the large- $r$ limit, this can be effectively regarded as a spatial compactification near the large M2-brane. ${ }^{6}$ From the structure of the metric (2.28), we find that the physical radius of the M-circle is given by $\tilde{R} \sim R /(\mu r)$. In the perspective of the type IIA superstring theory, the spherical M2-brane wrapping on the two-sphere in (2.28) corresponds to a D2-brane. The gauge coupling constant on D2-branes is given by $g_{Y M}^{2} \sim g_{s} l_{s}^{-1}$, where $g_{s}$ and $l_{s}$ are the string coupling and the string length. By translating this into the M-theory parameters using the standard dictionary, $g_{s} \sim\left(\tilde{R} / l_{p}\right)^{3 / 2}$ and $l_{s} \sim\left(l_{p}^{3} / \tilde{R}\right)^{1 / 2}$, one can express the coupling constant as $g_{Y M}^{2} \sim \tilde{R}^{2} / l_{p}^{3}$. In the limit where the radius of the $\mathrm{D}$-branes becomes large, it is convenient to rescale the metric, so that the parameter which controls the theory on D2-brane is given by the dimensionless coupling constant $g_{Y M}^{2} r_{\mathrm{M} 2}$, where $r_{\mathrm{M} 2}$ is the radius of the M2-brane. By using (2.19), The coupling constant can be expressed as

$$
g_{Y M}^{2} r_{\mathrm{M} 2} \sim \frac{R^{2}}{r_{\mathrm{M} 2} \mu^{2} l_{p}^{3}} .
$$

We are interested in the case where $l_{p}$ and $\mu$ are fixed. Moreover, in order to have an interacting theory on the D2-branes in the $r_{\mathrm{M} 2} \rightarrow \infty$ limit, we would like to fix the coupling constant (2.30). Then, the decoupling limit of the D2-branes is given by

$$
p^{+} \rightarrow \infty, \quad \frac{R^{2}}{p^{+}}: \text {fixed }
$$

\footnotetext{
${ }^{6}$ One can also take another coordinate $\left(\hat{x}^{+}, \hat{x}^{-}\right)$such that the metric becomes canonical Minkowski metric. In this coordinate, the both shifts of $\hat{x}^{ \pm}$are given by $R /(\mu r)$ and this looks like a light cone compactification. However, note that from $\frac{d}{d \hat{x}^{+}} \sim \frac{1}{\mu r} \frac{d}{d x^{+}}$, we see that the energy along $\hat{x}^{+}$direction is given by $\frac{1}{\mu r} H$. Similarly, the momentum along $\hat{x}^{-}$direction is $\mu r p^{+}+\frac{1}{\mu r} H$. In the limit discussed below, both $r$ and $p^{+}$becomes large, so that the energy is much smaller than the spatial momentum. Thus, after all, this can be indeed regarded as a spatial compactification.
} 
The fixed quantity in (2.31) measures the size of the M-circle for each fixed $p^{+}$, so that the M2-brane in 11-dimension is realized in the limit where $\frac{R^{2}}{p^{+}}$becomes large.

The limit (2.31) can be written in terms of the parameters of the matrix model. The D0-brane charge (the matrix size) $N$ is related to the light cone momentum by $p^{+}=N / R$ and the gauge coupling of D0-branes is given as $g^{2} \sim R^{3} l_{p}^{-6}$. Thus, the limit (2.31) is translated to

$$
N \rightarrow \infty, \quad \frac{g^{2}}{N}: \text { fixed. }
$$

Then, the decoupling limit of M2-brane is given by sending $\frac{g^{2}}{N}$ to infinity.

Next, we consider the decoupling limit of the spherical M5-brane. The theory on NS5-branes is known as the little string theory. This theory is characterized by the string tension proportional to $1 / l_{s}^{2}$. We can apply the above argument for D2-branes to the little string theory. Here, the fixed quantity is replaced by the tension of the little string which is made dimensionless by using the radius of the M5-brane (2.27):

$$
\frac{r_{\mathrm{M} 5}^{2}}{l_{s}^{2}} \sim \frac{\tilde{R} r_{\mathrm{M} 5}^{2}}{l_{p}^{3}} \sim \frac{R r_{\mathrm{M} 5}}{\mu l_{p}^{3}}
$$

Thus, the decoupling limit of NS5-brane is given by

$$
p^{+} \rightarrow \infty, \quad R^{4} p^{+}: \text {fixed }
$$

The M5-branes in 11-dimension are realized by further taking $R^{4} p^{+}$to be large.

In terms of the parameters of the matrix model, the decoupling limit of NS5brane (2.34) is translated into

$$
N \rightarrow \infty, \quad g^{2} N: \text { fixed. }
$$

This is just the 't Hooft limit of the matrix model. ${ }^{7}$ The M5-brane limit corresponds to the strong coupling limit with respect to the 't Hooft coupling $g^{2} N$.

For multiple M5-branes, the radius of each M5-brane should become large to decouple from the gravity. Furthermore, if there are some stacks of M5-branes with different radii as shown in figure 1, the distances between the nearest stacks should also become large. The limit realizing this situation is such that the all radii become large with the same order. Since the radius of each M5-brane is proportional to a positive power of the light cone momentum, the decoupling limit for the multiple fivebranes should be given by $(2.35)$ with $p_{s}^{+} / p_{t}^{+}$fixed for any $s, t=1, \cdots, \Lambda$. Thus, we find that the large- $N$ limit in (2.35) should be taken as in (1.4) in the case of the multiple M5-branes.

\section{The plane wave matrix model}

In this section, we review the plane wave matrix model (PWMM) $[2,9]$.

The Hamiltonian of PWMM is obtained by the matrix regularization of the Hamiltonian (2.14) of a single M2-brane [12]. In the matrix regularization, real functions on

\footnotetext{
${ }^{7}$ In [8], a possible logarithmic correction to this limit was found.
} 
the world volume $f\left(\sigma^{a}\right)$ are linearly mapped to $N \times N$ Hermitian matrices, in such a way that integrals and the Poisson algebra of functions are consistently mapped to traces and the commutator algebra of the corresponding matrices, respectively. Namely, under this mapping, we have

$$
\frac{1}{4 \pi} \int d^{2} \sigma \rightarrow \frac{1}{N} \operatorname{Tr}, \quad\{,\} \rightarrow \frac{-i N}{2}[,] .
$$

For example, let us consider the case where the spatial world volume is a unit sphere embedded in $R^{3}$. The image of the embedding function $x^{i}$ which satisfies (2.18) is given by the $N$-dimensional irreducible representation of the $\mathrm{SU}(2)$ generators,

$$
x_{i} \rightarrow \hat{x}_{i}=\frac{2}{N} L_{i}
$$

The normalization is chosen so that $\sum_{i} \hat{x}_{i}^{2}=1_{N}$ holds in the large- $N$ limit. One can check that (3.1) is satisfied by (3.2) for sufficiently large $N$.

By applying the matrix regularization to the Hamiltonian (2.14), we obtain the bosonic part of the Hamiltonian of $\mathrm{PWMM}^{8}$ as

$$
\begin{aligned}
H=\frac{4 \pi}{N} \operatorname{Tr} & {\left[\frac{4 \pi}{2 p^{+}}\left(\left(\frac{N}{4 \pi}\right)^{2} P_{A}^{2}-\frac{N^{2} T_{\mathrm{M} 2}^{2}}{8}\left[X_{A}, X_{B}\right]^{2}\right)\right.} \\
& \left.+\frac{p^{+}}{8 \pi}\left(\frac{\mu^{2}}{9} X_{i}^{2}+\frac{\mu^{2}}{36} X_{a}^{2}\right)+\frac{i \mu N T_{\mathrm{M} 2}}{12} \epsilon^{i j k} X_{i}\left[X_{j}, X_{k}\right]\right] .
\end{aligned}
$$

$P_{A}$ and $X_{A}(A=1,2, \cdots, 9)$ are now $N \times N$ matrices, which correspond to the images of $P_{A}\left(\sigma^{a}\right)$ and $X_{i}\left(\sigma^{a}\right)$ in (2.14). The constraint (2.15) is replaced by

$$
\left[P_{A}, X^{A}\right]=0 .
$$

In obtaining (3.3), we have also rescaled the momenta as $P_{A} \rightarrow\left(\frac{N}{4 \pi}\right) P_{A}$.

The rescaled momenta correspond to the canonical momenta of $X^{A}$ in PWMM. When one quantizes the theory of M2-brane (2.14), one has the canonical commutation relation, ${ }^{9}$

$$
\left[\hat{X}^{A}(\sigma), \hat{P}_{B}\left(\sigma^{\prime}\right)\right]=i \delta_{B}^{A} \delta^{(2)}\left(\sigma-\sigma^{\prime}\right) .
$$

Without the rescaling, according to (3.1), this would be mapped to

$$
\left[\hat{X}_{i j}^{A}, \hat{P}_{B k l}\right]=i \frac{N}{4 \pi} \delta_{B}^{A} \delta_{i l} \delta_{j k}
$$

The rescaling just removes the factor $\frac{N}{4 \pi}$ on the right-hand side and makes $\hat{P}_{A i j}$ the canonically normalized momenta of $\hat{X}_{i j}^{A}$.

We consider vacua of PWMM. Noticing that the potential for $X_{i}$ forms a perfect square, we find that the Hamiltonian is minimized when

$$
X^{i}=\frac{\mu p^{+}}{6 \pi N T_{\mathrm{M} 2}} L^{i}
$$

\footnotetext{
${ }^{8}$ See $[10,11]$ for BPS analysis of PWMM.

${ }^{9}$ Here, the commutator represents the commutator of operators acting on the Fock space and this should not be confused with the commutator of $N \times N$ matrices.
} 
and the other fields are equal to zero. Here, $L_{i}$ are $N$-dimensional representation matrices of the $\mathrm{SU}(2)$ generators. For any $N$-dimensional representation, (3.7) gives a vacuum of PWMM. ${ }^{10}$ In particular, the representation is reducible in general and we can make an irreducible decomposition to express $L_{i}$ as in (1.2). With this decomposition, the total matrix size can be written as $N=\sum_{s=1}^{\Lambda} N_{2}^{(s)} n_{s}$. Thus, the vacua of PWMM are labeled by the discrete moduli parameters, $\Lambda, N_{2}^{(s)}$ and $n_{s}$, which satisfy $N=\sum_{s=1}^{\Lambda} N_{2}^{(s)} n_{s}$.

For later convenience, we introduce the action of PWMM. We first rescale the matrices as

$$
Y^{A}=\frac{12 \pi N T_{\mathrm{M} 2}}{\mu p^{+}} X^{A}
$$

Then, the bosonic action of PWMM can be written in a simple form as ${ }^{11}$

$$
S=\frac{1}{g^{2}} \int d t \operatorname{Tr}\left[\frac{1}{2}\left(D Y^{A}\right)^{2}-2 Y_{i}^{2}-\frac{1}{2} Y_{a}^{2}+\frac{1}{4}\left[Y^{A}, Y^{B}\right]^{2}-i \epsilon_{i j k} Y^{i}\left[Y^{j}, Y^{k}\right]\right] .
$$

Here, the coupling constant is related to the original parameters by

$$
g^{2}=\frac{T_{\mathrm{M} 2}^{2}}{2 \pi}\left(\frac{12 \pi N}{\mu p^{+}}\right)^{3}
$$

and the covariant derivative is defined by

$$
D Y^{A}=\frac{\partial}{\partial t} Y^{A}-i\left[A, Y^{A}\right]
$$

The gauge field $A$ is introduced to take the constraint (3.4) into account. In the $A=0$ gauge, the Gauss law constraint reproduces (3.4).

\section{Spherical M5-branes from PWMM}

\subsection{Localization in PWMM}

We consider a complex scalar field in PWMM defined by

$$
\phi(t)=Y_{3}(t)+i\left(Y_{8}(t) \sin (t)+Y_{9}(t) \cos (t)\right) .
$$

The real and imaginary parts of $\phi$ are given by an $\mathrm{SO}(3)$ scalar and an $\mathrm{SO}(6)$ scalar, respectively, up to the time dependent rotation. When one makes a double Wick-rotation for the time and $Y_{9}$ directions, one can construct (four) supercharges which leave $\phi$ invariant. This allows us to exactly compute the expectation values of operators made of only $\phi$ by using the localization method [14].

In order to perform the localization, one first needs to define the boundary conditions in the Euclidean time direction. Since we are interested in PWMM expanded around a fixed vacuum, the appropriate boundary condition is such that all the fields approach to the vacuum configuration as the Euclidean time goes to $\pm \infty$. With this boundary condition, the path integral of PWMM defines the theory around the fixed background.

\footnotetext{
${ }^{10}$ See [13] for the relation of the vacua of PWMM to half-BPS states in the ABJM theory.

${ }^{11}$ We have also rescaled the time coordinate appropriately.
} 
For the theory around the generic vacuum (1.2), the result of the localization obtained in $[15-17]$ is summarized below. See appendix A for the detail of localization. We have the following equality:

$$
\left\langle\prod_{I} \operatorname{Tr} f_{I}\left(\phi\left(t_{I}\right)\right)\right\rangle=\left\langle\prod_{I} \operatorname{Tr} f_{I}\left(2 L_{3}+i M\right)\right\rangle_{M M}
$$

where $f_{I}(x)$ are arbitrary smooth functions, $2 L_{3}$ is the vacuum configuration for $Y_{3}$. The matrix $M$ in (4.2) is an $N \times N$ constant Hermitian matrix which commutes with all of $L_{a}(a=1,2,3)$. For the representation given by (1.2), $M$ takes the form,

$$
M=\bigoplus_{s=1}^{\Lambda}\left(\mathbf{1}_{n_{s}} \otimes M_{s}\right)
$$

where $M_{s}$ is an $N_{2}^{(s)} \times N_{2}^{(s)}$ Hermitian matrix. The expectation value $\langle\cdots\rangle$ on the left-hand side of (4.2) is taken with respect to the original action of PWMM expanded around the background (1.2). On the other hand, the expectation value $\langle\cdots\rangle_{M M}$ on the right-hand side of (4.2) is taken with respect to the following matrix integral:

$$
Z=\int \prod_{s=1}^{\Lambda} \prod_{i=1}^{N_{2}^{(s)}} d q_{s i} Z_{1-l o o p} e^{-\frac{2}{g^{2}} \sum_{s, i} n_{s} q_{s i}^{2}}
$$

where $q_{s i}\left(i=1,2, \cdots, N_{2}^{(s)}\right)$ are eigenvalues of $M_{s}$ and $Z_{1-\text { loop }}$ is the one-loop determinant, which arises in the 1-loop calculation of the localization. $Z_{1-\text { loop }}$ is given by

$$
Z_{1-\text { loop }}=\prod_{s, t=1}^{\Lambda} \prod_{J=\left|n_{s}-n_{t}\right| / 2}^{\left(n_{s}+n_{t}\right) / 2-1} \prod_{i=1}^{N_{2}^{(s)}} \prod_{j=1}^{N_{2}^{(t)}} '\left[\frac{\left\{(2 J+2)^{2}+\left(q_{s i}-q_{t j}\right)^{2}\right\}\left\{(2 J)^{2}+\left(q_{s i}-q_{t j}\right)^{2}\right\}}{\left\{(2 J+1)^{2}+\left(q_{s i}-q_{t j}\right)^{2}\right\}^{2}}\right]^{\frac{1}{2}}
$$

The prime on the last product means that the second factor in the numerator with $s=$ $t, J=0$ and $i=j$ is not included in the product.

Note that the right-hand side of (4.2) does not depend on the time coordinates $t_{a}$. So this relation implies that the correlator on the left-hand side does not depend on time. This property can be understood from the SUSY Ward identity, as shown in [16].

We remark that, in the calculation of the localization, some possible instanton corrections are neglected [15-17]. This corresponds to kink-like configurations in PWMM which connect two distinct vacua [18-21]. However, the instanton amplitudes are bounded from below by $N_{2} / \lambda$ times the difference of the quadratic Casimirs of the two vacua. Thus, in the decoupling limit of the M5-brane, this effect is suppressed.

\subsection{Coincident M5-branes from the simplest partition}

To illustrate our computation, let us first consider the simplest partition with $\Lambda=1$, namely the vacuum with

$$
L_{i}=L_{i}^{\left[N_{5}\right]} \otimes 1_{N_{2}} .
$$


According to the proposal in [3], this corresponds to $N_{5}$ coincident M5-branes. In this case, the eigenvalue integral (4.4) reduces to a one matrix model:

$$
Z=\int \prod_{i} d q_{i} \prod_{J=0}^{N_{5}-1} \prod_{i>j}^{N_{2}} \frac{\left\{(2 J+2)^{2}+\left(q_{i}-q_{j}\right)^{2}\right\}\left\{(2 J)^{2}+\left(q_{i}-q_{j}\right)^{2}\right\}}{\left\{(2 J+1)^{2}+\left(q_{i}-q_{j}\right)^{2}\right\}^{2}} e^{-\frac{2 N_{5}}{g^{2}} \sum_{i} q_{i}^{2}}
$$

In the decoupling limit of the M5-brane, $N_{2}$ becomes infinity, so that the saddle point approximation is valid in evaluating the eigenvalue integral (4.7). As usual, we introduce the eigenvalue distribution

$$
\rho(q)=\frac{1}{N_{2}} \sum_{i=1}^{N_{2}} \delta\left(q-q_{i}\right)
$$

which is normalized as

$$
\int_{-q_{m}}^{q_{m}} d q \rho(q)=1
$$

Here, $q_{m}$ represents the range of the support of $\rho(x) .{ }^{12}$ Note that, we are interested in the decoupling limit of M5-brane where the 't Hooft coupling $\lambda:=g^{2} N_{2}$ goes to infinity. In this regime, the Gaussian attractive force of the eigenvalue integral (4.7) becomes weaker, so that $q_{m}$ is expected to go to infinity. If one considers the region where $q_{m}$ is very large compared to $N_{5}$, one can reduce the saddle point equation of $\rho(x)$ to

$$
\beta=\pi \rho(q)+\frac{2 N_{5}}{\lambda} q^{2}-\int d q^{\prime} \frac{2 N_{5}}{\left(2 N_{5}\right)^{2}+\left(q-q^{\prime}\right)^{2}} \rho\left(q^{\prime}\right),
$$

where $\beta$ is the Lagrange multiplier, which imposes the normalization (4.9). See appendix B for the derivation of (4.10)

In the M5-brane limit, the solution to the saddle point equation is given by

$$
\rho(q)=\frac{8^{3 / 4}}{3 \pi \lambda^{1 / 4}}\left[1-\frac{q^{2}}{q_{m}^{2}}\right]^{3 / 2}, \quad q_{m}=(8 \lambda)^{1 / 4}, \quad \beta=\frac{8^{1 / 2} N_{5}}{\lambda^{1 / 2}} .
$$

See appendix C.1 for the derivation of this solution. ${ }^{13}$ Note that indeed $q_{m}$ becomes infinity as the 't Hooft coupling goes to infinity.

By using this solution, we can compute correlation functions of $\phi$. For example,

$$
\begin{aligned}
\frac{1}{N}\left\langle\operatorname{Tr} \phi^{2}(0)\right\rangle & =\frac{1}{N}\left\langle\operatorname{Tr} Y_{3}^{2}(0)\right\rangle-\frac{1}{N}\left\langle\operatorname{Tr} Y_{9}^{2}(0)\right\rangle=\frac{1}{N}\left\langle\operatorname{Tr}\left(2 L_{3}+i M\right)^{2}\right\rangle_{M M} \\
& =\frac{1}{N} 4 \operatorname{Tr}\left(L_{3}^{2}\right)-\frac{1}{N}\left\langle\operatorname{Tr} M^{2}\right\rangle_{M M} \\
& =\frac{N_{5}^{2}-1}{12}-\int_{-q_{m}}^{q_{m}} d q q^{2} \rho(q) \\
& =\frac{N_{5}^{2}-1}{12}-\frac{\sqrt{8 \lambda}}{6} .
\end{aligned}
$$

Note that the second term is much larger than the first term in the strong coupling regime with $N_{5}$ fixed. This originally comes from the fact that the eigenvalue distribution of $M$

\footnotetext{
${ }^{12}$ Note that $\rho$ has a single support, because the potential in (4.7) has a single well.

${ }^{13}$ See also [16] for another derivation using the Fermi gas method.
} 
spreads over the much wider region than the distribution of $L_{3}$ in the M5-brane limit. This property is common for any correlation function of $\phi$, including the resolvent. In this regime, therefore, the imaginary part of $\phi$ is dominant and the real part is negligible. In other words, the spectrum of $\phi$ lies along the imaginary axis in this limit.

Assuming that the matrices $Y^{A}$ become mutually commuting in the decoupling limit, one may expect that this spectrum on the imaginary axis given by $\rho$ in (4.11) could be identified with the eigenvalue distribution of one of the $\mathrm{SO}(6)$ scalars. However, such identification would contradict with the discussion in [22] by Polchinski. In [22], the BFSS matrix model is considered and the trace of the square of the scalar fields $Y^{A}$ is shown to be bounded from below by $\lambda^{2 / 3}$ (in the notation used in this paper). And this conclusion is considered to hold also for PWMM if we assume the gauge/gravity correspondence: the dual geometry of PWMM reduces to the dual geometry of BFSS matrix model at a sufficiently large radius $r \gg \mathcal{O}\left(\lambda^{1 / 4}\right)$ in the decoupling limit of M5-brane [23]. On the other hand, if one assumes that $\rho$ in (4.11) gives the eigenvalue distribution of one of the $\mathrm{SO}(6)$ scalars in PWMM, this would give $\frac{1}{N} \operatorname{Tr}\left(Y^{A}\right)^{2}=\mathcal{O}\left(\lambda^{1 / 2}\right)$, which is smaller than the bound in the Polchinski's argument. Thus, this leads to a contradiction and the first assumption that $Y_{A}$ become commuting in the decoupling limit seems to be wrong. ${ }^{14}$

Apart from Polchinski's argument, we can find another reasoning for the above statement, based on the gauge/gravity correspondence. The gravity dual [23] of PWMM has a typical scale $\lambda^{1 / 3}$, which is the string scale beyond which the supergravity approximation is not valid. It is natural to expect that the matrix elements of $Y^{A}$ contain information of such typical scale on the gravity side, so that the scalar fields in PWMM have the typical value $\frac{1}{N} \operatorname{Tr}\left(Y^{A}\right)^{2}=\mathcal{O}\left(\lambda^{2 / 3}\right)$. Then, it is again suggested that the matrices are noncommuting even in the strongly coupled region.

The classical geometry of the supergravity and the M2/M5-branes are considered to be realized as the low energy moduli of these matrices. Roughly speaking, they will correspond to the low energy modes of the matrices and one needs to consider the low energy theory of the matrix model to find the classical geometric objects in M-theory. The noncommuting modes, which produce the large value for $\frac{1}{N} \operatorname{Tr}\left(Y^{A}\right)^{2}=\mathcal{O}\left(\lambda^{2 / 3}\right)$, have a large excitation energy, so that these modes should be frozen and irrelevant in studying the low energy theory.

Note that the complex field $\phi$ has the eigenvalue distribution of order of $\lambda^{1 / 4}$. This is much smaller than the typical value of the noncommuting modes. From this fact, we find that $\phi$ is a good low energy field and the operators $\operatorname{Tr} \phi^{n}$ can be considered as operators in the low energy theory. This can also be understood from our formula (4.2) of the localization. The correlation functions of $\phi$ are independent of the time coordinates and hence are invariant under taking the time averages, which projects the operators to the low energy modes (More specifically, one can eliminate the high energy modes by integrating over very short time intervals with length given by $1 / C$, where $C$ is a constant much smaller than the typical energy scale for noncommuting modes but much larger than the energy scale for (4.11).). This means that the result of the localization (4.2) contains only the low energy modes.

\footnotetext{
${ }^{14}$ We thank J. Maldacena for suggesting this problem and the resolution using the time average which we will discuss below.
} 
As is discussed in [22], operators in the matrix model should be additively renormalized in the low energy theory, where the additive renormalization constants correspond to contributions from the high energy noncommuting modes. However, such additive renormalization is not needed for $\operatorname{Tr} \phi^{n}$. In order for the eigenvalues of $\phi$ to be of $\mathcal{O}\left(\lambda^{1 / 4}\right)$, the renormalization constants for $Y^{A}$ must cancel out in the correlators of $\phi$. For example, this can be seen in our computation in (4.12). Since the $\operatorname{Tr} \phi^{2}$ is given by the difference between $\operatorname{Tr}\left(Y^{3}\right)^{2}$ and $\operatorname{Tr}\left(Y^{9}\right)^{2}$, the renormalization constants should cancel out.

The statement that $\phi$ picks up the low energy moduli of the matrices is also supported by the earlier work on the gauge/gravity correspondence for PWMM. It was shown in [16, 17] that the field $\phi$ describes a system of moduli parameters on the gravity side, which is equivalent to a certain axially symmetric electrostatic system: the charge densities of the electrostatic system, which determines the geometry on the gravity side, were shown to be equivalent to the eigenvalue density of $\phi$.

From these observations, we claim that the spectrum of $\phi$ is identified with the low energy moduli of PWMM. Furthermore, we claim that the low energy moduli in PWMM are given by commuting matrices in the decoupling limit. This can be understood as follows. Suppose that the moduli are given by noncommuting matrices and the theory on the M5-branes has some noncommutativity of the low energy moduli parameters as well as some length scale associated with the noncommutativity. The noncommutative length scale must be much smaller than the radius of the M5-brane, since otherwise the M5-brane would not be localized along the radial direction due to the nonlocality caused by the noncommutativity and hence would not be regarded as $1+5$ dimensional object. Then, let us consider the length scale $\lambda^{1 / 4}$ of the low energy moduli computed from the localization. This scale corresponds to the scale of the M5-brane radius if one takes the rescaling (3.8) into account. Thus, the length scale of the low energy moduli must be much larger than the noncommutative scale. Therefore, even if the moduli have noncommutativity, this effect must be much smaller than the value of the moduli themselves in the decoupling limit. Thus, we can ignore the noncommutativity and can regard the moduli as just commuting matrices. Note that this conclusion is consistent with our result of the localization (4.2). Here, the moduli distribution is given by the distribution of $2 L_{3}+i M$ in (4.7), and $L_{3}$ and $M$ are indeed mutually commuting variables.

The commutativity of the low energy moduli matrices might be general phenomena which occur in the strong coupling limit. As observed in [24], in some matrix models with commutator interactions, commuting matrices indeed arise in the strong coupling limit. A possible mechanism is as follows. For Yang-Mills type matrix models, one can rescale the matrices in such a way that the coupling constant appears in front of each commutators. In the strong coupling limit, in order to have a finite value of the action, the values of commutators themselves must become small unless there is some cancellation with the kinetic terms. If this occurs, the matrices become commuting with each other. Though observing this phenomena directly in the current model is very difficult, this is very likely to occur in the low energy region, since in the low energy limit, the kinetic terms of the matrices are very small and there will be no chance to have a cancellation between the kinetic terms and the commutator terms. 
Thus, we identify the real and imaginary parts of $\phi$ in the formula (4.2) with the low energy moduli for $Y^{3}$ and $Y^{9}$, respectively. In particular, $\rho$ in (4.11) is identified with the moduli of $Y^{9}$. Recall that, in the decoupling limit of M5-brane, we have seen that the spectrum of $\phi$ becomes pure imaginary. Hence, with the suitable normalization of matrices (namely, going back to the original normalization in (3.8)), one finds that the moduli of the $\mathrm{SO}(6)$ scalar have a wide distribution while the moduli of the $\mathrm{SO}(3)$ scalars collapse to the origin in the decoupling limit of the M5-brane.

Now, let us consider the description of the spherical M5-brane. We consider the $\mathrm{SO}(6)$ symmetric uplift of the distribution $[25,26]$ of the moduli of a single $\mathrm{SO}(6)$ scalar. The uplifted distribution $\tilde{\rho}$ is defined as the solution of

$$
\int d^{6} x \tilde{\rho}(r) x_{9}^{2 n}=\left(\frac{\mu p^{+}}{12 \pi N T_{\mathrm{M} 2}}\right)^{2 n} \int_{-q_{m}}^{q_{m}} d q \rho(q) q^{2 n},
$$

for any $n$, where $r=\sqrt{x_{a}^{2}}$ is the distance from the origin. The normalization factor on the right-hand side is chosen so that $\tilde{\rho}$ represents a density function before the rescaling (3.8). For the density $\rho$ in (4.11), the unique solution to (4.13) is

$$
\tilde{\rho}(r)=\frac{1}{V_{5} r_{0}^{5}} \delta\left(r-r_{0}\right) .
$$

The radius $r_{0}$ is given by

$$
r_{0}=\left(\frac{\mu p^{+}}{6 \pi^{3} N_{5} T_{\mathrm{M} 5}}\right)^{1 / 4}
$$

For $N_{5}=1$, the shape of the density function of the $\mathrm{SO}(6)$ moduli agrees with the shape of the spherical M5-brane. In particular, the radius shows a perfect agreement with the M5-brane: $r_{0}=r_{\mathrm{M} 5}$. Therefore, we conclude that the spherical M5-brane is indeed realized as the low energy moduli distribution of the $\mathrm{SO}(6)$ scalar fields in PWMM.

For $N_{5}>1,(4.14)$ should correspond to the radius of multiple coincident M5-branes. The $N_{5}$-dependence of the radius agrees with the expected form in [3] based on the perturbative expansion in PWMM.

\subsection{Multiple M5-branes from generic partitions}

Let us generalize the above calculation to the case of the general partition (1.2). According to [3], this corresponds to $\Lambda$ stacks of M5-branes with different radii as shown in figure 1 . As we discussed in section 2.3, to make the M5-branes decouple from the bulk gravity, we consider the limit (2.35) such that the large- $N$ limit is taken as in (1.4).

We introduce the eigenvalue distribution for $q_{s i}$ in (4.4) for each $s$ as

$$
\rho_{s}(q)=\sum_{i=1}^{N_{2}^{(s)}} \delta\left(q-q_{s i}\right)
$$

and again assume that $\rho_{s}(q)$ has a single support $\left[-q_{s}, q_{s}\right]$. Note that, to simplify some expressions below, here we use the normalization

$$
\int_{-q_{s}}^{q_{s}} \rho_{s}(q)=N_{2}^{(s)}
$$


which is different from the one we used in the previous subsection. The saddle point equations for $\rho_{s}(q)$ can be derived in the same way as (4.10) and take the form,

$\rho_{s}(q)+\frac{1}{\pi} \sum_{t=1}^{\Lambda} \int_{-q_{t}}^{q_{t}} d u\left\{\frac{\left|n_{s}-n_{t}\right|}{\left|n_{s}-n_{t}\right|^{2}+(u-q)^{2}}-\frac{n_{s}+n_{t}}{\left(n_{s}+n_{t}\right)^{2}+(u-q)^{2}}\right\} \rho_{t}(u)=\frac{\mu_{s}}{\pi}-\frac{2 n_{s}}{\pi g^{2}} q^{2}$,

where $q \in\left[-q_{s}, q_{s}\right]$ and $s=1,2, \cdots, \Lambda$. In appendix C.2, we construct a solution to these equations in the decoupling limit. The solution is given as

$$
\hat{\rho}_{s}(q)=\frac{8^{3 / 4} \sum_{r=1}^{s} N_{2}^{(r)}}{3 \pi \lambda_{s}^{1 / 4}}\left[1-\frac{q^{2}}{q_{r}^{2}}\right]^{\frac{3}{2}}, \quad q_{s}=\left(8 \lambda_{s}\right)^{1 / 4}, \quad \lambda_{s}:=g^{2} \sum_{r=1}^{s} N_{2}^{(r)},
$$

where $s=1,2, \cdots, \Lambda$ and $\hat{\rho}_{s}(q)$ are defined by

$$
\hat{\rho}_{s}(q):=\sum_{r=1}^{s} \rho_{r}(q)
$$

The variables $\hat{\rho}_{s}(q)(s=1,2, \cdots, \Lambda)$ have the following properties. First, $\hat{\rho}_{s}(q)$ is defined on the interval $\left[-q_{s}, q_{s}\right]$ and is normalized as

$$
\int_{q_{s}}^{q_{s}} d q \hat{\rho}_{s}(q)=\sum_{r=1}^{s} N_{2}^{(s)}
$$

Note that $\sum_{r=1}^{s} N_{2}^{(s)}$ is proportional to the light cone momentum of the M5-brane in the $s$ th stack (1.3). Second, $\hat{\rho}_{s}(q)$ naturally appear in evaluating the correlation functions of the complex field $\phi$. As we discussed in the previous subsection, in the decoupling limit of M5-branes, $L_{i}$ on the right-hand side in (4.2) can be ignored. Then, we have for example,

$$
\begin{aligned}
\left\langle\operatorname{Tr} \phi^{n}\right\rangle & =i^{n}\left\langle\operatorname{Tr} M^{n}\right\rangle_{M M}=i^{n} \sum_{s=1}^{\Lambda} \sum_{i=1}^{N_{2}^{(s)}} n_{s}\left\langle q_{s i}^{n}\right\rangle_{M M} \\
& =i^{n} \int d q q^{n}\left[n_{1} \rho_{1}(q)+n_{2} \rho_{2}(q)+\cdots+n_{\Lambda-1} \rho_{\Lambda-1}(q)+n_{\Lambda} \rho_{\Lambda}(q)\right] \\
& =i^{n} \int d q q^{n}\left[\left(n_{1}-n_{2}\right) \hat{\rho}_{1}(q)+\left(n_{2}-n_{3}\right) \hat{\rho}_{2}(q)+\cdots+\left(n_{\Lambda-1}-n_{\Lambda}\right) \hat{\rho}_{\Lambda-1}(q)+n_{\Lambda} \hat{\rho}_{\Lambda}(q)\right] .
\end{aligned}
$$

Note that the coefficient $\left(n_{s}-n_{s+1}\right)$ of $\hat{\rho}_{s}$ is just the number of M5-branes in the $s$ th stack. From these properties, $\hat{\rho}_{s}$ can be naturally identified with the density function for an M5-brane in the $s$ th stack.

Obviously, the $\mathrm{SO}(6)$ symmetric uplift of $\left\{\hat{\rho}_{s}\right\}$ is given by $\Lambda$ stacks of the spherical shells. By taking the rescaling (3.8) into account, the sth stack has the radius

$$
r_{s}=\frac{q_{s} \mu}{12 \pi R T_{\mathrm{M} 2}}=\left(\frac{\mu p_{s}^{+}}{6 \pi^{3} T_{M_{5}}}\right)^{1 / 4},
$$

where $p_{s}^{+}$is defined in (1.3). Thus, we have shown that, as shown in figure 1 , the generic partition indeed describes concentric stacks of M5-branes with radii given by (4.23). 


\section{Spherical M2-branes from PWMM}

So far, we considered the description of M5-branes in PWMM. In this section, we apply the same analysis to the M2-brane limit. Note that the emergence of the spherical D2-branes in the type IIA superstring theory can be understood even at the level of the classical action. However, it still nontrivial whether we can observe the emergence in the strong coupling region of PWMM. Here, we study the emergence of M2-branes in the decoupling limit of the M2-branes. In this section, we only consider the simplest partition (4.6) but the generalization is straightforward.

In the M2-brane limit, where $N_{5}$ goes to infinity, the one-loop determinant of the eigenvalue integral (4.7) converges to the hyperbolic tangent function. Thus, we obtain

$$
Z=\int \prod_{i} d q_{i} \prod_{i>j}^{N_{2}} \tanh ^{2}\left(\frac{\pi\left(q_{i}-q_{j}\right)}{2}\right) e^{-\frac{2 N_{5}}{g^{2}} \sum_{i} q_{i}^{2}}
$$

Note that the model depends only on $N_{2}$ and $g^{2} / N_{5}$.

The typical value of the eigenvalues of this model should depend on $N_{2}$ and $g^{2} / N_{5}$. Then, in the decoupling limit of M2-branes, the typical value is much smaller than $N_{5}$. This implies that, in the result of the localization (4.2), the eigenvalue distribution of $M$ is much narrower than that of $L_{3}$. Hence the spectrum of $\phi$ lies on the real axis in this limit. This implies that the moduli of $Y^{3}$ are given by the classical vacuum configuration $2 L_{3}$ while the moduli of $Y^{9}$ collapse to the origin. It is easy to see that the $\mathrm{SO}(3)$ symmetric uplift of this configuration gives the two-sphere and the radius agrees with that of the spherical M2-brane on the supergravity side for $N_{2}=1$. Thus, we see that the spherical M2-brane is also realized as the moduli of $\mathrm{SO}(3)$ scalars.

However, we should notice that, unlike the decoupling limit of M5-branes, the instanton corrections could contribute to the partition function in the M2-brane limit. ${ }^{15}$ If this is the case, since the result of the localization does not include the instanton corrections [15-17], our computation is not correct. Then, in order for our computation to make sense, we need to consider the limit where the number of M2-branes goes to infinity. In this limit, the instanton effects will be suppressed. Thus, at least in the large- $N_{2}$ case, the result of the localization shows the emergence of the spherical M2-branes in PWMM.

When $N_{2}$ is large, we can find an exact solution for the eigenvalue distribution of (5.1) and can check that the typical value of the eigenvalues is proportional to $\left(\lambda / N_{5}\right)^{1 / 3}$ for large $\lambda / N_{5}$. See appendix D.

Of course, there is still a possibility that the instantons do not affect our computation. For instance, this happens if there exists a fermionic zero mode at the saddle points of instantons in the localization computation. This needs a further analysis of the localization saddles in PWMM.

\footnotetext{
${ }^{15}$ This effect can naturally be understood as the instantons on the theory on D2-branes, which connect two vacua with different monopole charges [3].
} 


\section{Summary and discussion}

In this paper, we tested a conjecture on the description of spherical M5-branes in the matrix model formulation of M-theory. We considered the plane wave matrix model (PWMM), which is expected to describe the M-theory on the maximally supersymmetric 11-dimensional plane wave geometry.

We first reviewed that, in the M-theory, there exist spherical M2- and M5- branes with zero light cone energy. These spherical branes are considered to be described as certain vacuum states in PWMM. This relation between the spherical branes and the vacua of PWMM is stated in [3]. In particular, it is conjectured that a single spherical M5-brane corresponds to the trivial vacuum of PWMM.

Through a direct computation in PWMM using the localization, we showed that the spherical M2- and M5- branes are formed by the distribution of the moduli of $\mathrm{SO}(3)$ and $\mathrm{SO}(6)$ scalar fields, respectively. This result strongly supports the proposal in [3].

As we discussed in section 4.2, we can assume that the moduli in PWMM are given by commuting matrices in the decoupling limit of the M5-branes. Here, let us consider a possible effective theory of these commuting matrices in the decoupling limit. We require the theory to have the $\mathrm{SO}(6)$ symmetry and to be able to reproduce our result of the localization. For the case of coincident M5-branes, a possible solution to these requirements is given by a commuting matrix model with 6 matrices defined by ${ }^{16}$

$$
\hat{S}=N^{2}\left[\frac{m^{2}}{2} \int d^{6} \overrightarrow{y^{\prime}} \hat{\rho}\left(\overrightarrow{y^{\prime}}\right) \vec{y}^{2}-\int d^{6} \vec{y} d^{6} \overrightarrow{y^{\prime}} \hat{\rho}(\vec{y}) \hat{\rho}\left(\overrightarrow{y^{\prime}}\right) \ln \left|\vec{y}-\overrightarrow{y^{\prime}}\right|-\beta\left(\int d^{6} \overrightarrow{y^{\prime}} \hat{\rho}\left(\overrightarrow{y^{\prime}}\right)-1\right)\right],
$$

where $\hat{\rho}$ is the distribution of moduli $y_{i}^{a}(a=4,5, \cdots, 9, i=1,2, \cdots, N)$ for the $\mathrm{SO}(6)$ scalars $Y^{a}$,

$$
\hat{\rho}(y)=\frac{1}{N} \sum_{i=1}^{N} \delta^{(6)}\left(\vec{y}_{i}-\vec{y}\right) .
$$

The Lagrange multiplier $\beta$ is introduced to impose the normalization condition on $\hat{\rho}$. The second term in (6.1) is understood as the Vandermonde determinant $\prod_{i<j}\left|\vec{y}_{i}-\vec{y}_{j}\right|^{2}$ for the commuting matrices. We fix the parameter $m$ in (6.1) as

$$
m=(8 \lambda)^{-\frac{1}{4}}
$$

so that the model reproduces the result of the localization below. In the 't Hooft limit, the WKB approximation becomes exact. The saddle point equation is given by

$$
\beta=\frac{m^{2}}{2} \vec{y}^{2}-\int d^{6} \overrightarrow{y^{\prime}} \hat{\rho}\left(\overrightarrow{y^{\prime}}\right) \ln \left|\vec{y}-\overrightarrow{y^{\prime}}\right|^{2} .
$$

The solution to this equation is obtained in [26-28] as

$$
\hat{\rho}(\vec{y})=\frac{1}{\pi^{3}|\vec{y}|^{5}} \delta\left(|\vec{y}|-\frac{1}{m}\right) .
$$

\footnotetext{
${ }^{16}$ The same model was also considered in different contexts [26-28].
} 
Note that, through the rescaling (3.8), this is indeed equivalent to (4.14) obtained from the localization. Thus, the saddle point configuration of the commuting matrix model agrees with the configuration of the coincident spherical M5-branes. This agreement suggests that the commuting matrix model might be relevant to a certain sector of the low energy theory of PWMM.

It would be interesting to find more general commuting matrix model, which reproduces our result for the general partition. In addition, we also need to investigate whether some low energy excitations can also be reproduced from the commuting matrix model or not.

Finding a good description of the low energy theory should be one of the most important problem in understanding the description of the classical geometry in the matrix theory. We hope that our result gives a clue to this problem.

\section{Acknowledgments}

We thank D. O'Connor, D. Berenstein, J. Maldacena, H. Shimada and K. Shimizu for valuable discussions. Y. A. was supported by a Dublin Institute for Advanced Studies scholarship. The work of G. I. was supported, in part, by Program to Disseminate Tenure Tracking System, MEXT, Japan and by KAKENHI (16K17679). S. S. was supported by the MEXT-Supported Program for the Strategic Research Foundation at Private Universities Topological Science (Grant No. S1511006). The work of S. T. was supported by JSPS KAKENHI Grant Number 17K05414.

\section{A Localization in PWMM}

In this appendix, we perform the localization and derive the formula (4.2). In this appendix, following the method in [14], we use a Lorentzian signature obtained by a double Wick rotation for the time-direction and the direction of one of the $\mathrm{SO}(6)$ scalar fields. To use some 10 dimensional notation, we relabel the $\mathrm{SO}(3)$ scalar fields in PWMM as $\left(Y_{1}, Y_{2}, Y_{3}\right) \rightarrow$ $\left(Y_{2}, Y_{3}, Y_{4}\right)$, the $\mathrm{SO}(6)$ scalar fields as $\left(Y_{4}, \cdots, Y_{9}\right) \rightarrow\left(Y_{5}, \cdots, Y_{10}\right)$ and the gauge field as $A \rightarrow Y_{1}$. The double Wick rotation is performed for the $Y_{1}$ and $Y_{10}$ directions and hence, the $Y_{1}$ 's direction is Euclidean and $Y_{10}$ 's direction is Lorentzian. We also use $Y_{0}$ to express the scalar field in the Lorentzian signature, which is related to $Y_{10}$ by $Y_{0}=i Y_{10}$.

\section{A.1 Off-shell supersymmetry of PWMM}

In the above notation, the full action of PWMM can be written in the 10-dimensional notation as

$$
S_{P W}=\frac{1}{g^{2}} \int d \tau \operatorname{Tr}\left(\frac{1}{4} \sum_{M, N=1}^{10} F_{M N} F^{M N}+\frac{1}{2} \sum_{a=5}^{10} Y_{a} Y^{a}+\frac{i}{2} \sum_{M=1}^{10} \Psi \Gamma^{M} D_{M} \Psi\right),
$$


Here, $\Psi$ is the 10-dimensional Majorana Weyl spinor with 16 components and we use the gamma matrices defined in [15]. We have also used the following notation:

$$
\begin{aligned}
F_{1 M} & =D_{1} Y_{M}=\partial_{\tau} Y_{M}-i\left[Y_{1}, Y_{M}\right] \quad(M \neq 1), \\
F_{i j} & =2 \varepsilon_{i j k} Y_{k}-i\left[Y_{i}, Y_{j}\right], \quad F_{i a}=D_{i} Y_{a}=-i\left[Y_{i}, Y_{a}\right], \quad F_{a b}=-i\left[Y_{a}, Y_{b}\right], \\
D_{1} \Psi & =\partial_{\tau} \Psi-i\left[Y_{1}, \Psi\right], \quad D_{i} \Psi=\frac{1}{4} \varepsilon_{i j k} \Gamma^{j k} \Psi-i\left[Y_{i}, \Psi\right], \quad D_{a} \Psi=-i\left[Y_{a}, \Psi\right],
\end{aligned}
$$

where $i, j, k=2,3,4$ and $a, b=5,6, \cdots, 10$. In order to realize the off-shell supersymmetries, we further add seven auxiliary fields

$$
-\frac{1}{g^{2}} \int d \tau \frac{1}{2} \sum_{I=1}^{7} \operatorname{Tr} K_{I} K_{I}
$$

to the action (A.1). Under the Wick rotation, $K_{I}$ shall become anti-Hermitian, so that (A.3) becomes positive definite in the Euclidean signature.

The theory has the off-shell supersymmetry,

$$
\begin{aligned}
\delta_{s} Y_{M} & =-i \Psi \Gamma_{M} \epsilon, \\
\delta_{s} \Psi & =\frac{1}{2} F_{M N} \Gamma^{M N} \epsilon-Y_{a} \tilde{\Gamma}^{a} \Gamma^{19} \epsilon+K^{I} \nu_{I}, \\
\delta_{s} K_{I} & =i \nu_{I} \Gamma^{M} D_{M} \Psi .
\end{aligned}
$$

See [15] for the definition of $\tilde{\Gamma}^{a}$. The parameter $\epsilon$ has to satisfy the Killing spinor equation of PWMM and the closure of the supersymmetry requires $\nu_{I}$ to satisfy

$$
\begin{aligned}
\epsilon \Gamma^{M} \nu_{I} & =0, \\
\frac{1}{2}\left(\epsilon \Gamma_{N} \epsilon\right) \tilde{\Gamma}_{\alpha \beta}^{N} & =\nu_{\alpha}^{I} \nu_{\beta}^{I}+\epsilon_{\alpha} \epsilon_{\beta}, \\
\nu_{I} \Gamma^{M} \nu_{J} & =\delta_{I J} \epsilon \Gamma^{M} \epsilon .
\end{aligned}
$$

The following spinors give a solution to these conditions:

$$
\epsilon=e^{\frac{\tau}{2} \Gamma^{09}} e^{-\frac{\pi}{4} \Gamma^{49}}\left(\begin{array}{c}
\eta_{1} \\
0 \\
0 \\
0
\end{array}\right), \quad \nu_{I}=\sqrt{2} e^{\frac{\tau}{2} \Gamma^{09}} e^{-\frac{\pi}{4} \Gamma^{49}} \Gamma^{I 8}\left(\begin{array}{c}
\eta_{1} \\
0 \\
0 \\
0
\end{array}\right),
$$

where $\eta_{1}$ is any 4 -component constant vector. We use $\eta_{1}=(1,0,0,0)$ in the following computation.

\section{A.2 Saddle point of the localization}

To perform the localization, we add an exact term $t \delta_{s} V$ to the action, where

$$
V=\int d \tau \operatorname{Tr} \Psi \overline{\delta_{s} \Psi}
$$


After some calculation, one can find that the bosonic part of $\delta_{s} V$ is calculated to be

$$
\begin{aligned}
\delta_{s} V \sim & -e^{\tau}\left(D_{1} Y_{0}+Y_{0}-e^{-\tau} K_{5}\right)^{2}-e^{-\tau}\left(D_{1} Y_{0}-Y_{0}+e^{\tau} K_{5}\right)^{2}-2 c \sum_{i=2}^{4}\left(D_{i} Y_{0}\right)^{2} \\
& -2 c \sum_{I \neq 5}\left(K^{I}\right)^{2}+2 c\left(D_{4} Y_{9}\right)^{2}+2 c\left[Y_{0}, Y_{9}\right]^{2}+2 c \sum_{a=5}^{8}\left[Y_{0}, Y_{a}\right]^{2}+\mathcal{S} \\
& +4 \sum_{a=1}^{3}\left[e^{-\tau}\left\{F_{a 4}^{+}-\frac{1}{2} D_{a}\left(e^{\tau} Y_{9}\right)+F_{a+4,8}^{+}\right\}^{2}+e^{\tau}\left\{F_{a 4}^{-}+\frac{1}{2} D_{a}\left(e^{-\tau} Y_{9}\right)-F_{a+4,8}^{-}\right\}^{2}\right],
\end{aligned}
$$

where $c:=\cosh \tau$ and $\mathcal{S}$ is defined by

$$
\begin{aligned}
\mathcal{S}= & e^{\tau}\left(Y_{5}+D_{1} Y_{5}+D_{2} Y_{6}+D_{3} Y_{7}+D_{4} Y_{8}+e^{-\tau} F_{98}\right)^{2} \\
& +e^{-\tau}\left(Y_{5}-D_{1} Y_{5}-D_{2} Y_{6}-D_{3} Y_{7}+D_{4} Y_{8}-e^{\tau} F_{98}\right)^{2} \\
& +e^{\tau}\left(Y_{6}+D_{1} Y_{6}-D_{2} Y_{5}+D_{3} Y_{8}-D_{4} Y_{7}-e^{-\tau} F_{97}\right)^{2} \\
& +e^{-\tau}\left(Y_{6}-D_{1} Y_{6}+D_{2} Y_{5}-D_{3} Y_{8}-D_{4} Y_{7}+e^{\tau} F_{97}\right)^{2} \\
& +e^{\tau}\left(Y_{7}+D_{1} Y_{7}-D_{2} Y_{8}-D_{3} Y_{5}+D_{4} Y_{6}+e^{-\tau} F_{96}\right)^{2} \\
& +e^{-\tau}\left(Y_{7}-D_{1} Y_{7}+D_{2} Y_{8}+D_{3} Y_{5}+D_{4} Y_{6}-e^{\tau} F_{96}\right)^{2} \\
& +e^{\tau}\left(Y_{8}+D_{1} Y_{8}+D_{2} Y_{7}-D_{3} Y_{6}-D_{4} Y_{5}-e^{-\tau} F_{95}\right)^{2} \\
& +e^{-\tau}\left(Y_{8}-D_{1} Y_{8}-D_{2} Y_{7}+D_{3} Y_{6}-D_{4} Y_{5}+e^{\tau} F_{95}\right)^{2} .
\end{aligned}
$$

The derivatives $D_{M}$ are defined in (A.2). $F_{a b}^{ \pm}$stands for the selfdual and anti-selfdual part of $F_{a b}^{ \pm}$in the subspace $a, b=1,2,3,4$ or $a, b=5,6,7,8$. After the Wick rotation, $Y_{0}=i Y_{10}$ and $K_{i}=i K_{i}^{(E)}(i=1,2, \cdots, 7)$, the bosonic part $\left.\delta_{s} V\right|_{b o s}$ becomes a sum of positive-definite terms.

We consider the theory around a fixed vacuum (1.2). Then, we impose the boundary condition such that all fields approach to the vacuum configuration at $\tau \rightarrow \pm \infty$. Then, taking the temporal gauge $Y_{1}=0$, we find that the saddle point configuration is given by

$$
\hat{Y}_{10}=\frac{M}{c}, \quad \hat{K}_{5}^{(E)}=\frac{M}{c^{2}}, \quad \hat{Y}_{i}=-2 L_{i-1}(i=2,3,4),
$$

where all the other fields are zero. Here, $2 L_{i}(i=1,2,3)$ are the vacuum configuration and $M$ is a constant Hermitian matrix, which commutes with all of $L_{i}$. For the vacuum of the form (1.2), $M$ takes the form (4.3).

It is easy to see that the gaussian part in (4.4) is obtained by substituting the saddle point configuration to the classical action $S_{P W}$. The remaining part $Z_{1-\text { loop }}$ in (4.4) is obtained by the 1-loop calculation around the saddle point.

\section{A.3 Ghost fields}

We introduce the collective notation,

$$
X=\left(\begin{array}{c}
Y_{A} \\
(\epsilon \epsilon) \Upsilon_{I}
\end{array}\right), \quad X^{\prime}=\left(\begin{array}{c}
-i(\epsilon \epsilon) \Psi_{A} \\
H_{I}
\end{array}\right)
$$


where $\Upsilon_{I}, H_{I}(I=1,2, \cdots, 7)$ and $\Psi_{A}(A=1, \cdots, 9)$ are defined below. Since $\left\{\Gamma^{A} \epsilon, \nu_{I} \mid A=1, \cdots, 9, I=1, \cdots, 7\right\}$ gives an orthogonal basis for 16 component spinors, $\Psi$ can be expanded as

$$
\Psi=\Psi_{A} \Gamma^{A} \epsilon+\Upsilon_{I} \nu^{I}
$$

$\Psi_{A}$ and $\Upsilon_{I}$ are introduced as the coefficients of this expansion. $H_{I}$ are defined as

$$
H_{I}=(\epsilon \epsilon) K_{I}+2 \nu_{I} \tilde{\epsilon} Y_{0}+\nu_{I}\left(\frac{1}{2} \sum_{A, B=1}^{9} F_{A B} \Gamma^{A B} \epsilon-2 \sum_{a=5}^{9} X_{a} \Gamma^{a} \tilde{\epsilon}\right)
$$

where $\tilde{\epsilon}=\frac{1}{2} \Gamma^{19} \epsilon$. We also define

$$
\phi=Y_{0} \cosh \tau-Y_{4}+Y_{9} \sinh \tau .
$$

Then, the supersymmetry can be written as

$$
\delta_{s} X=X^{\prime}, \quad \delta_{s} X^{\prime}=-i\left(\delta_{\phi}+\delta_{\mathrm{U}(1)}\right) X, \quad \delta_{s} \phi=0,
$$

where $\delta_{\phi}$ is a gauge transformation with the parameter given by $\phi$ and $\delta_{\mathrm{U}(1)}$ is a diagonal $\mathrm{U}(1)$ transformation of the $\mathrm{SO}(3) \times \mathrm{SO}(6)$ symmetry. This shows that $X$ and $X^{\prime}$ forms a doublet while $\phi$ is a singlet under the supersymmetry.

We also introduce the ghost fields, $\left(C, C_{0}, \tilde{C}, \tilde{C}_{0}, b, b_{0}, a_{0}, \tilde{a}_{0}\right)$, where $\left(b, b_{0}, a_{0}, \tilde{a}_{0}\right)$ are bosonic and $\left(C, \tilde{C}, C_{0}, \tilde{C}_{0}\right)$ are fermionic fields. The fields with subscript 0 shall contain only zero modes for both $\tau$ direction and the fuzzy sphere directions. They are defined through the following BRS transformations,

$$
\begin{aligned}
\delta_{B} X & =[X, C], & \delta_{B} X^{\prime} & =\left[X^{\prime}, C\right], \\
\delta_{B} C & =a_{0}-C^{2}, & \delta_{B} \phi & =[\phi, C], \\
\delta_{B} \tilde{C} & =b, & \delta_{B} b & =\left[\tilde{C}, a_{0}\right], \\
\delta_{B} \tilde{a}_{0} & =i \tilde{C}_{0}, & \delta_{B} \tilde{C}_{0} & =-i\left[\tilde{a}_{0}, a_{0}\right], \\
\delta_{B} b_{0} & =i C_{0}, & \delta_{B} C_{0} & =-i\left[b_{0}, a_{0}\right],
\end{aligned}
$$

The commutator in the above equation shall express the anti-commutator for fermionic variables. The square of $\delta_{B}$ is a gauge transformation with parameter $a_{0}$,

$$
\delta_{B}^{2}=\left[, a_{0}\right]
$$

We define the supersymmetry transformation of the ghost fields as

$$
\delta_{s} C=\phi, \quad \delta_{s}(\text { the other ghosts })=0 .
$$

Then $Q=\delta_{s}+\delta_{B}$ has the following action:

$$
\begin{aligned}
Q X & =X^{\prime}+[X, C], & Q X^{\prime} & =-i\left(\delta_{\phi}+\delta_{\mathrm{U}(1)}\right) X+\left[X^{\prime}, C\right], \\
Q C & =\phi+a_{0}-C^{2}, & Q \phi & =[\phi, C], \\
Q \tilde{C} & =b, & Q b & =\left[\tilde{C}, a_{0}\right], \\
Q \tilde{a}_{0} & =i \tilde{C}_{0}, & Q \tilde{C}_{0} & =-i\left[\tilde{a}_{0}, a_{0}\right], \\
Q b_{0} & =i C_{0}, & Q C_{0} & =-i\left[b_{0}, a_{0}\right],
\end{aligned}
$$


One can easily show that $Q^{2}$ is given as

$$
Q^{2}=-i \delta_{\mathrm{U}(1)}+\left[, a_{0}\right] .
$$

The gauge-fixing and ghost actions are defined by

$$
S_{g h}=\int d \tau Q \operatorname{Tr}\left[i \tilde{C}\left(F+b_{0}\right)+C \tilde{a}_{0}\right],
$$

where $F$ corresponds to the gauge fixing condition. We use

$$
F=\sum_{a=1}^{4} \hat{D}_{a}\left(\frac{1}{\cosh \tau} Y_{a}\right)
$$

for our computation, where the background covariant derivative $\hat{D}_{a}$ is defined by

$$
\hat{D}_{a} X:=-i\left[\hat{Y}_{a}, X\right] \quad(a=1,2,3,4) .
$$

Here, $\hat{Y}_{1}=i \frac{\partial}{\partial \tau}$ and $\hat{Y}_{i}(i=2,3,4)$ are the vacuum configuration of $Y_{i}$.

\section{A.4 1-loop determinants}

Let us perform the 1-loop calculation around the saddle point (A.10). We first redefine the fields as

$$
\tilde{X}^{\prime}:=X^{\prime}+[X, C], \quad \tilde{\phi}:=2 \phi+a_{0}-C^{2},
$$

and divide the fields to four groups as

$$
\begin{array}{ll}
Z_{0}=\left(Y_{A}, \tilde{a}_{0}, b_{0}\right), & Z_{1}=\left(\Upsilon_{I}, C, \tilde{C}\right), \\
Z_{0}^{\prime}=\left(\tilde{\Psi}_{A}, \tilde{C}_{0}, C_{0}\right), & Z_{1}^{\prime}=\left(\tilde{H}_{I}, \tilde{\phi}, b\right) .
\end{array}
$$

They form doublets under the action of $Q$ as

$$
Q Z_{i}=Z_{i}^{\prime}, \quad Q Z_{i}^{\prime}=R Z_{i}, \quad(i=0,1)
$$

where $R:=Q^{2}$ is given by the sum of the $\mathrm{U}(1)$ and gauge transformations as shown in (A.20).

Then we expand the full action $S_{P W}+t Q\left(V+V_{g h}\right)$ around the saddle point configuration (A.10) as $Z_{i} \rightarrow \hat{Z}_{i}+Z_{i}$ and $Z_{i}^{\prime} \rightarrow \hat{Z}^{\prime}{ }_{i}+Z_{i}^{\prime}$. Then the quadratic part of the fluctuations in $V+V_{g h}$ is schematically written as

$$
V^{(2)}=\left(Z_{0}^{\prime}, Z_{1}\right)\left(\begin{array}{cc}
D_{00} & D_{01} \\
D_{10} & D_{11}
\end{array}\right)\left(\begin{array}{c}
Z_{0} \\
Z_{1}^{\prime}
\end{array}\right)
$$

where $D_{i j}(i, j=0,1)$ are some linear differential operators. Thus, the quadratic part of the action takes the form

$$
Q V^{(2)}=\left(R Z_{0}, Z_{1}^{\prime}\right)\left(\begin{array}{cc}
D_{00} & D_{01} \\
D_{10} & D_{11}
\end{array}\right)\left(\begin{array}{c}
Z_{0} \\
Z_{1}^{\prime}
\end{array}\right)+\left(Z_{0}^{\prime}, Z_{1}\right)\left(\begin{array}{cc}
D_{00} & D_{01} \\
D_{10} & D_{11}
\end{array}\right)\left(\begin{array}{c}
Z_{0}^{\prime} \\
R Z_{1}
\end{array}\right) .
$$


Hence, the one-loop integral produces the determinants,

$$
Z_{1-\text { loop }}=\left(\frac{\operatorname{det}_{V_{Z_{1}}} R}{\operatorname{det}_{V_{Z_{0}}} R}\right)^{\frac{1}{2}} .
$$

Here, the determinants should be taken in the appropriate functional spaces of the fluctuations. Recall that we adopted the boundary condition such that all fields go to the vacuum configuration as $\tau \rightarrow \infty$. This implies that the fluctuations should vanish at infinities.

Note that $D_{10}$ is a linear map from $V_{Z_{0}}$ to $V_{Z_{1}}$ and commutes with $R$. Then the determinants in (A.29) cancel between $\operatorname{Im} D_{10} \subset V_{Z_{1}}$ and $\operatorname{Im} D_{10}^{*} \subset V_{Z_{0}}$, where $D_{10}^{*}$ is the adjoint of $D_{10}$. Hence, the 1-loop determinant reduces to

$$
Z_{1-\text { loop }}=\left(\frac{\operatorname{det}_{\operatorname{coker} D_{10} R} R}{\operatorname{det}_{\operatorname{ker} D_{10}} R}\right)^{\frac{1}{2}} .
$$

Furthermore, since $R$ and $D_{10}$ commute, the kernel and the cokernel are given by direct sums of the eigenspaces of $R$. Thus, we can express the 1-loop determinant as

$$
Z_{1-\text { loop }}=\prod_{i} r_{i}^{\left(\operatorname{dim} V_{r_{i}}^{\prime}-\operatorname{dim} V_{r_{i}}\right) / 2}
$$

where $V_{r_{i}}$ and $V_{r_{i}}^{\prime}$ are the restrictions of the kernel and the cokernel to the eigenspace of $R$ with eigenvalue $r_{i}$, respectively. Therefore, the remaining task is to evaluate $r_{i}$ and the index $\operatorname{dim} V_{r_{i}}^{\prime}-\operatorname{dim} V_{r_{i}}$ in each eigenspace.

By integrating the ghost field $\tilde{a}_{0}$, we obtain the constraint $a_{0}=-2 \phi$. At the saddle point, this is equal to $-2 i M+4 L_{4}$. Thus, $r_{i}$ is given by the sum of eigenvalues of $[-2 i M+$ $\left.4 L_{4}, \quad\right]$ and the diagonal $\mathrm{U}(1)$ charge.

By studying the structure of $D_{10}$ for each supersymmetry multiplet, we can easily compute the index. The result is as follows [15]. The contribution from the hypermultiplet, which contains $Y_{5}, \cdots, Y_{8}$, is given by

$$
\prod_{s, t=1}^{\Lambda} \prod_{J=\left|n_{s}-n_{t}\right| / 2}^{\left(n_{s}+n_{t}\right) / 2-1} \prod_{i=1}^{N_{2}^{(s)}} \prod_{j=1}^{N_{2}^{(t)}} \frac{1}{(2 J+1)^{2}+\left(q_{s i}-q_{t j}\right)^{2}}
$$

The contribution from the vector multiplet, which contains $Y_{1}, \cdots, Y_{4}, Y_{9}$, is given by

$$
\begin{aligned}
& \prod_{s, t=1}^{\Lambda} \prod_{\substack{J=\left|n_{s}-n_{t}\right| / 2 \\
J \neq 0}}^{\left(n_{s}+n_{t}\right) / 2-1} \prod_{i=1}^{N_{2}^{(s)}} \prod_{j=1}^{N_{2}^{(t)}}\left\{(2 J)^{2}+\left(q_{s i}-q_{t j}\right)^{2}\right\}^{1 / 2} \\
& \quad \times \prod_{s, t=1}^{\Lambda} \prod_{J=\left|n_{s}-n_{t}\right| / 2}^{\left(n_{s}+n_{t}\right) / 2-1} \prod_{i=1}^{N_{2}^{(s)}} \prod_{j=1}^{N_{2}^{(t)}}\left\{(2 J+2)^{2}+\left(q_{s i}-q_{t j}\right)^{2}\right\}^{1 / 2} .
\end{aligned}
$$

Combining these contributions with the Vandermonde determinant for diagonalizing $M$, we obtain the 1-loop determinant (4.5). See below for the derivation of these 1-loop determinants. 


\section{A.5 Derivation of 1-loop determinants}

The relevant part of the action is given by $Z_{1} D_{10} Z_{0}$. In terms of the component fields, this can be written explicitly as

$$
\begin{aligned}
& 2 s_{i} \Upsilon_{i}+i \tilde{C}\left(F+b_{0}\right)+C \tilde{a}_{0} \\
& -\frac{i}{\epsilon \epsilon}\left(\delta_{\mathrm{U}(1)} Y_{A}-2 i\left[\hat{Y}_{A}, v^{4} Y_{4}+v^{9} Y_{9}\right]-i\left[Y_{A},-2 i M+v^{4} \hat{Y}_{4}\right]\right)\left[\hat{Y}_{A}, C\right],
\end{aligned}
$$

where

$$
s_{i}:=\nu_{i}\left(\frac{1}{2} \sum_{A, B=1}^{9} F_{A B} \Gamma^{A B} \epsilon-2 \sum_{a=5}^{9} X_{a} \Gamma^{a} \tilde{\epsilon}\right) .
$$

Note that the fields in the hypermultiplet, $\left\{\left(Y_{m}, \Upsilon_{i}\right) \mid m=5,8,7,8, i=1,2,3,4\right\}$, decouple from the fields in the vector multiplet in (A.34). Hence, the index has two independent contributions from these two sectors.

Index theorem in 1-dimension. For the computation of the 1-loop determinant, the index theorem in 1-dimension is very useful, which we will describe below.

The setup is as follows. We consider the set of all $n$-dimensional vector valued smooth functions on $R$ vanishing at infinity, $S:=\left\{f: R \rightarrow C^{n} \mid \lim _{\tau \rightarrow \pm \infty} f(\tau)=0\right\}$. Let us introduce a linear differential operator $D$ on $S$ as

$$
D f(\tau):=\frac{\partial f}{\partial \tau}(\tau)+(A \cdot f)(\tau)
$$

where $f \in S$ and $A: R \rightarrow M_{n}(C)$. $A \cdot f$ is just the standard action of matrices, $(A \cdot f)_{i}(\tau):=$ $A_{i j}(\tau) f_{j}(\tau)$. For the computation of the 1-loop determinant, we only consider the case where $A$ is bounded at both infinities as $\lim _{\tau \rightarrow \pm \infty} A_{i j}(\tau)<\infty(i, j=1, \cdots, n)$ and $A(\tau)$ is diagonalizable as

$$
V^{-1}(\tau) A(\tau) V(\tau)=A_{d}(\tau):=\operatorname{diag}\left(\lambda_{1}(\tau), \cdots, \lambda_{n}(\tau)\right) .
$$

As $A$ is bounded, both of $\lim _{\tau \rightarrow \pm \infty} A_{i j}(\tau)$ and $\lim _{\tau \rightarrow \pm \infty} \lambda_{i}(\tau)$ are some constants. Then, $\lim _{\tau \rightarrow \pm \infty} V(\tau)$ are also constant matrices.

The 1-dimensional index theorem follows from the fact that the number of positive and negative eigenvalues of $A$ at both infinities determines the index of $D$. The essential statement of the index theorem is that if the $k(1 \leq k \leq n)$ eigenvalues in (A.37) satisfy both

$$
\lim _{\tau \rightarrow \infty} \operatorname{Re} \lambda_{i}(\tau)>0 \text { and } \lim _{\tau \rightarrow-\infty} \operatorname{Re} \lambda_{i}(\tau)<0
$$

and the remaining $n-k$ eigenvalues do not, then, we have

$$
\operatorname{dim}(\operatorname{ker} D)=k \text {. }
$$

This relation can be shown as follows. Note that $D$ is covariant under $A \rightarrow U^{-1} A U+U^{-1} \partial U$. Consider the gauge transformation such that

$$
U^{-1} A U+U^{-1} \partial U=A_{d}
$$


Such $U$ can be expressed as $U(\tau)=\left[P \exp \left(-\int^{\tau} A\right)\right] \exp \left(\int^{\tau} A_{d}\right)$, where $P$ denotes the path ordering. The general solution to the differential equation $D f=0$ is then given by

$$
f(\tau)=U(\tau) \exp \left(-\int_{0}^{\tau} A_{d}\left(\tau^{\prime}\right) d \tau^{\prime}\right) f_{0},
$$

where $f_{0}$ is a constant vector. In order to be a solution in the space of $S$, (A.41) has to vanish at both infinities. Here, let us consider the condition (A.38). When $k$ of $\lambda_{i}$ 's satisfy (A.38), only $k$ components of $f_{0}$ can be nonzero to satisfy the boundary conditions. This implies (A.39).

Of course, the similar equation to (A.39) holds for the adjoint operator $D^{\dagger}$. By combining this with (A.39), we obtain the index theorem in 1-dimension, which states that the index of $D$ is completely determined by the behavior of $A$ at infinities.

Hypermultiplet. Let us consider the hypermultiplet. We use complex combinations,

$$
W_{1}=Y_{5}+i Y_{8}, \quad W_{2}=Y_{6}+i Y_{7}
$$

We can read off the action of $D_{10}$ from (A.34). If $\left(W_{1}, W_{2}\right)$ is an element of $\operatorname{ker} D_{10}$, we have

$$
\begin{aligned}
& \partial W_{1}+2 i\left[L_{-}, W_{2}\right]+\frac{s}{c}\left(W_{1}+2\left[L_{3}, W_{1}\right]\right)=0, \\
& \partial W_{2}-2 i\left[L_{+}, W_{1}\right]+\frac{s}{c}\left(W_{2}-2\left[L_{3}, W_{2}\right]\right)=0,
\end{aligned}
$$

where $s=\sinh \tau$ and $c=\cosh \tau$. To analyze the structure of these equation, we use the fuzzy spherical harmonics, which behave nicely under the adjoint action of $L_{i}$. See [29-31] for the definition. For the vacuum of the form (1.2) we can decompose $W_{i}(i=1,2)$ to the block components $\left\{W_{i}^{(s, t)} \mid s, t=1,2, \cdots, \Lambda\right\}$. We then expand each block with the fuzzy spherical harmonics $\hat{Y}_{J m\left(j_{s}, j_{t}\right)}$ as

$$
W_{i}^{(s, t)}=\sum_{J=\left|j_{s}-j_{t}\right|}^{j_{s}+j_{t}} \sum_{m=-J}^{J} W_{i J m}^{(s, t)} \otimes \hat{Y}_{J m\left(j_{s}, j_{t}\right)} . \quad(i=1,2)
$$

Then, (A.43) becomes

$$
\begin{aligned}
& \partial W_{1 J m}^{(s, t)}+\frac{s}{c}(1+2 m) W_{1 J m}^{(s, t)}+2 i \delta_{-} W_{2 J m+1}^{(s, t)}=0, \\
& \partial W_{2 J m}^{(s, t)}+\frac{s}{c}(1-2 m) W_{2 J m}^{(s, t)}-2 i \delta_{+} W_{1 J m-1}^{(s, t)}=0,
\end{aligned}
$$

where $\delta_{ \pm}=\sqrt{(J \pm m)(J \mp m+1)}$. It is easy to check that (A.38) is satisfied only by $W_{1 J J}^{(s, t)}$ and $W_{2 J-J}^{(s, t)}$. Indeed, these modes have eigenvalues $(2 J+1) \tanh \tau$ which satisfy (A.38). Thus, only $W_{1 J J}^{(s, t)}$ and $W_{2 J-J}^{(s, t)}$ and their complex conjugates contribute to the index.

Then, let us consider the contribution from fermions, $\left\{\Upsilon_{i}, i=1,2,3,4\right\}$. We introduce complex fields as

$$
\xi_{1}=\Upsilon_{1}+i \Upsilon_{4}, \quad \xi_{2}=\Upsilon_{3}+i \Upsilon_{2}
$$


and expand their block components by the spherical harmonics as we did above. Then, we can obtain

$$
\begin{aligned}
& \partial \xi_{1 J m}^{(s, t)}+\frac{2 s m}{c} \xi_{1 J m}^{(s, t)}+2 \delta_{+} \xi_{2 J m-1}^{(s, t)}=0 \\
& \partial \xi_{2 J m}^{(s, t)}-\frac{2 s m}{c} \xi_{2 J m}^{(s, t)}+2 \delta_{-} \xi_{1 J m+1}^{(s, t)}=0
\end{aligned}
$$

for $\xi_{1}, \xi_{2} \in \operatorname{coker} D_{10}$. In this case, there is no eigenvalue satisfying (A.38). Hence, these modes have no contribution to the index.

Thus, we find that only $W_{1 J J}^{(s, t)}$ and $W_{2 J-J}^{(s, t)}$ and their complex conjugates contribute to the index. The eigenvalues of $R$ for these modes are $r=2\left( \pm(2 J+1)+i\left(q_{s i}-q_{t j}\right)\right)$, and thus we obtain (A.32).

Vector multiplet. Next, we consider the vector multiplet. We first calculate $\operatorname{dim}\left(\operatorname{ker} D_{10}\right)$. For $\left\{Y_{A}, \tilde{a}_{0}, b_{0} \mid A=1,2,3,4,9\right\} \in \operatorname{ker} D_{10}$, we have

$$
\begin{aligned}
F+b_{0} & =0, \\
\tilde{a}_{0}+2\left[\hat{Y}_{A}, \frac{1}{\epsilon \epsilon}\left[\hat{Y}_{A}, v^{4} Y_{4}+v^{9} Y_{9}\right]\right]+\left[\left[\hat{Y}_{A}, \frac{1}{\epsilon \epsilon} Y_{A}\right],-2 i M+v^{4} \hat{Y}_{4}\right] & =0, \\
c\left(2 Y_{4}-i\left[\hat{Y}_{2}, Y_{3}\right]+i\left[\hat{Y}_{3}, Y_{2}\right]\right)-s\left(\partial Y_{4}+i\left[\hat{Y}_{4}, Y_{1}\right]\right)-\partial Y_{9} & =0, \\
c\left(\partial Y_{3}+i\left[\hat{Y}_{3}, Y_{1}\right]\right)-s\left(2 Y_{3}+i\left[\hat{Y}_{2}, Y_{4}\right]-i\left[\hat{Y}_{4}, Y_{2}\right]\right)-i\left[\hat{Y}_{2}, Y_{9}\right] & =0, \\
c\left(\partial Y_{2}+i\left[\hat{Y}_{2}, Y_{1}\right]\right)-s\left(2 Y_{2}-i\left[\hat{Y}_{3}, Y_{4}\right]+i\left[\hat{Y}_{4}, Y_{3}\right]\right)+i\left[\hat{Y}_{3}, Y_{9}\right] & =0 .
\end{aligned}
$$

To simplify the equations, let us consider the limit $\tau \rightarrow \pm \infty$ in (A.48). Since $F \rightarrow 0$ in this limit, we obtain $b_{0}=0$. Noticing that $b_{0}$ has only the constant mode, by using (A.48) again, we find that $F$ should be vanishing for arbitrary point on $R$, namely,

$$
F=\sum_{a=1}^{4}\left[\hat{Y}_{a}, \frac{1}{\cosh \tau} Y_{a}\right]=0
$$

Similarly, $\tilde{a}_{0}=0$ follows from (A.49). By substituting these vanishing conditions to (A.49), we obtain,

$$
-\partial\left(\frac{1}{c} \partial\left(Y_{4}-s Y_{9}\right)\right)+\frac{4}{c} \sum_{i=1}^{3}\left[L_{i},\left[L_{i}, Y_{4}-s Y_{9}\right]\right]=0 .
$$

This equation implies $Y_{4}-s Y_{9}=0$ as follows. Putting $f=Y_{4}-s Y_{9}$, the equation (A.54) has the form $\partial^{2} f-\frac{s}{c} \partial f-4 J(J+1) f=0$, where $J(J+1)$ is the eigenvalue of $\left[L_{i},\left[L_{i},\right]\right]$. From the boundary condition, $f / c$ should vanish at infinity. Then, it follows that

$$
0=\int d \tau \partial\left(\frac{1}{c^{2}} f \partial f\right)=\int d x\left[\left(\frac{\partial f}{c}\right)^{2}+\left(\frac{4 J(J+1)-1}{c^{2}}+\frac{3}{2 c^{4}}\right) f^{2}\right]
$$

For $J \neq 0$, the right-hand side is a sum of positive definite terms and hence $f$ itself must be zero. For $J=0$, the equation (A.54) is just $\partial((\partial f) / c)=0$. By integrating this equation under the boundary condition $f / c \rightarrow 0$, we find that $f$ is constant. We then 
consider (A.50) with $f$ constant. From this equation, we can easily obtain $Y_{4}=Y_{9}=0$ for $J=0$. Therefore, the relation $Y_{4}=s Y_{9}$ holds for any $J$.

Then, by eliminating $Y_{4}$ by $Y_{4}=s Y_{9}$, the equations (A.48), (A.50), (A.51), (A.52) become

$$
\begin{aligned}
-i \partial Y_{1}+i \frac{s}{c} Y_{1}+\left[L_{+}, Y_{-}\right]+\left[L_{-}, Y_{+}\right]+2 s\left[L_{3}, Y_{9}\right] & =0, \\
-\left[L_{+}, Y_{-}\right]+\left[L_{-}, Y_{+}\right]+s Y_{9}-c \partial Y_{9}+2 i \frac{s}{c}\left[L_{3}, Y_{1}\right] & =0, \\
c\left(\partial Y_{+}-2 i\left[L_{+}, Y_{1}\right]\right)-s\left(2 Y_{+}-2\left[L_{3}, Y_{+}\right]\right)-2 c^{2}\left[L_{+}, Y_{9}\right] & =0, \\
c\left(\partial Y_{-}-2 i\left[L_{-}, Y_{1}\right]\right)-s\left(2 Y_{-}+2\left[L_{3}, Y_{-}\right]\right)+2 c^{2}\left[L_{-}, Y_{9}\right] & =0,
\end{aligned}
$$

where $Y_{ \pm}=Y_{2} \pm i Y_{3}$.

We then make a redefinition $Y_{9}^{\prime}=c Y_{9},{ }^{17}$ and expand each block component of $Y_{ \pm}, Y_{1}, Y_{9}^{\prime}$ by the fuzzy spherical harmonics. For $f=$ $\left(Y_{J m+1}^{+(s, t)} / \sqrt{2}, Y_{J m-1}^{-(s, t)} / \sqrt{2}, i Y_{J m}^{1(s, t)}, Y_{J m}^{\prime 9(s, t)}\right)^{T}(m=-J+1,-J+2, \cdots, J-1, J \geq 1)$, the equations (A.56) take the same form as (A.36), where $A$ is given by

$$
A=\left(\begin{array}{cccc}
\frac{2 m s}{c} & 0 & -\sqrt{2} \delta_{-} & -\sqrt{2} \delta_{-} \\
0 & -\frac{2 m s}{c} & -\sqrt{2} \delta_{+} & \sqrt{2} \delta_{+} \\
-\sqrt{2} \delta_{-} & -\sqrt{2} \delta_{+} & -\frac{s}{c} & -\frac{2 m s}{c} \\
-\sqrt{2} \delta_{-} & \sqrt{2} \delta_{+} & -\frac{2 m s}{c} & -\frac{2 s}{c}
\end{array}\right) .
$$

This matrix does not have any eigenvalues, which satisfy (A.38). Hence, we find that the bosonic fields in the vector multiplet do not contribute to the index.

Let us apply the same analysis to the fermions. For $\left(C, \tilde{C}, \Upsilon_{5}, \Upsilon_{6}, \Upsilon_{7}\right) \in \operatorname{coker} D_{10}$, we have

$$
\begin{array}{r}
-\frac{1}{c} \partial \tilde{C}+\frac{1}{c}\left[i M-2 L_{3}, \partial C\right]-8 s\left[L_{3}, \Upsilon_{5}\right]-8 c\left[L_{2}, \Upsilon_{6}\right]+8 c\left[L_{1}, \Upsilon_{7}\right]=0, \\
\frac{1}{c}\left[L_{1}, \tilde{C}\right]-\frac{1}{c}\left[L_{1},\left[i M-2 L_{4}, C\right]\right]+4 i c\left[L_{2}, \Upsilon_{5}\right]-4 i s\left[L_{3}, \Upsilon_{6}\right]-2 c \partial \Upsilon_{7}-6 s \Upsilon_{7}=0, \\
\frac{1}{c}\left[L_{2}, \tilde{C}\right]-\frac{1}{c}\left[L_{2},\left[i M-2 L_{4}, C\right]\right]-4 i c\left[L_{1}, \Upsilon_{5}\right]-4 i s\left[L_{3}, \Upsilon_{7}\right]+2 c \partial \Upsilon_{6}+6 s \Upsilon_{6}=0, \\
\frac{1}{c}\left[L_{3}, \tilde{C}\right]+\partial\left(\frac{1}{c} \partial C\right)-\frac{4}{c} \sum_{i=1}^{3}\left[L_{i},\left[L_{i}, C\right]\right]-\frac{1}{c}\left[L_{3},\left[i M-2 L_{3}, C\right]\right]+2 s \partial \Upsilon_{5}+6 c \Upsilon_{5} \\
+4 i s\left[L_{1}, \Upsilon_{6}\right]+4 i s\left[L_{2}, \Upsilon_{7}\right]=0, \\
-s \partial\left(\frac{1}{c} \partial C\right)+\frac{4 s}{c} \sum_{i=1}^{3}\left[L_{i},\left[L_{i}, C\right]\right]+2 \partial \Upsilon_{5}+4 i\left[L_{1}, \Upsilon_{6}\right]+4 i\left[L_{2}, \Upsilon_{7}\right]=0 .
\end{array}
$$

We make some redefinitions as $\tilde{C}^{\prime}=\left(\tilde{C}-\left[i M-2 L_{4}, C\right]\right) /(2 \sqrt{2} c), C^{\prime}=C / c, \Upsilon_{5}^{\prime}=\sqrt{2} \Upsilon_{5}$ and

\footnotetext{
${ }^{17}$ Note that $Y_{9}^{\prime}$ does not necessarily vanish at infinities. However, only when $Y_{9}^{\prime}$ vanishes at infinities, (A.56) has nontrivial solutions. So we assume that $Y_{9}^{\prime} \rightarrow 0$ as $\tau \rightarrow \pm \infty$.
} 
also introduce complex fields, $\Upsilon_{ \pm}=\Upsilon_{6} \pm i \Upsilon_{7}$. With this notation, we can write (A.58) as

$$
\begin{array}{r}
\partial C^{\prime}-d=0, \\
\partial d+\frac{3 s}{c} d+2 C^{\prime}-4 \sum_{i=1}^{3}\left[L_{i},\left[L_{i}, C^{\prime}\right]\right]+\frac{2 \sqrt{2}}{c^{2}}\left[L_{3}, \tilde{C}^{\prime}\right]+\frac{3 \sqrt{2}}{c^{2}} \Upsilon_{5}^{\prime}=0, \\
\partial \Upsilon_{+}-\sqrt{2} i\left[L_{+}, \tilde{C}^{\prime}\right]-\sqrt{2} i\left[L_{+}, \Upsilon_{5}^{\prime}\right]+\frac{3 s}{c} \Upsilon_{+}-\frac{2 s}{c}\left[L_{3}, \Upsilon_{+}\right]=0, \\
\partial \Upsilon_{-}+\sqrt{2} i\left[L_{-}, \tilde{C}^{\prime}\right]-\sqrt{2} i\left[L_{-}, \Upsilon_{5}^{\prime}\right]+\frac{3 s}{c} \Upsilon_{-}+\frac{2 s}{c}\left[L_{3}, \Upsilon_{-}\right]=0, \\
\partial \tilde{C}^{\prime}+\frac{2 s}{c} \tilde{C}^{\prime}+\frac{2 s}{c}\left[L_{3}, \Upsilon_{5}^{\prime}\right]-\sqrt{2} i\left(\left[L_{+}, \Upsilon_{-}\right]-\left[L_{-}, \Upsilon_{+}\right]\right)=0, \\
\partial \Upsilon_{5}^{\prime}+\frac{2 s}{c}\left[L_{3}, \tilde{C}^{\prime}\right]+\frac{3 s}{c} \Upsilon_{5}^{\prime}+\sqrt{2} i\left(\left[L_{+}, \Upsilon_{-}\right]+\left[L_{-}, \Upsilon_{+}\right]\right)=0,
\end{array}
$$

where a new field $d$ is introduced to make the equations first order.

We then expand each block component by fuzzy spherical harmonics. For $f=$ $\left({C^{\prime}}_{J m}^{(s, t)}, d_{J m}^{(s, t)}, \Upsilon_{J m+1}^{+(s, t)}, \Upsilon_{J m-1}^{-(s, t)}, \Upsilon_{J m}^{\prime 5(s, t)}, \tilde{C}_{J m}^{\prime(s, t)}\right)^{T}(m=-J+1,-J+2, \cdots, J-1)$, the above equation can be written in the form of (A.36), where

$$
A=\left(\begin{array}{cccccc}
0 & -1 & 0 & 0 & 0 & 0 \\
\frac{3 s}{c} 2-4 J(J+1) & 0 & 0 & \frac{3 \sqrt{2}}{c^{2}} & \frac{3 \sqrt{2} m}{c^{2}} \\
0 & 0 & \frac{s}{c}(1-2 m) & 0 & -\sqrt{2} i \delta_{-} & -\sqrt{2} i \delta_{-} \\
0 & 0 & 0 & \frac{s}{c}(1+2 m) & -\sqrt{2} i \delta_{+} & \sqrt{2} i \delta_{+} \\
0 & 0 & \sqrt{2} i \delta_{-} & \sqrt{2} i \delta_{+} & \frac{3 s}{c} & \frac{2 m s}{c} \\
0 & 0 & \sqrt{2} i \delta_{-} & -\sqrt{2} i \delta_{+} & \frac{2 m s}{c} & \frac{2 s}{c}
\end{array}\right)
$$

It is easy to see that there is no eigenvalues of $A$ that satisfy (A.38). Hence, these modes have no contribution to the index.

On the other hand, the highest momentum modes $f=$ $\left(C_{J J}^{\prime(s, t)}, d_{J J}^{(s, t)}, \Upsilon_{J J-1}^{-(s, t)}, \Upsilon_{J J}^{\prime 5(s, t)}, \tilde{C}_{J J}^{\prime(s, t)}\right)^{T}$ have a nontrivial contribution. They satisfy (A.36) where $A$ is given by a $5 \times 5$ matrix obtained by eliminating the fifth row and column (namely, those for $\Upsilon_{+}$) and putting $m=J$ in (A.60). Then, we can find that there is just one eigenvalue which satisfies (A.38). In the same way, we can see that the modes with $m=-J$ have the same structure. ${ }^{18}$ The eigenvalues of $R$ for these modes are $r=2\left( \pm 2 J+i\left(q_{s i}-q_{t j}\right)\right)$. This contribution gives the first line of (A.33).

Finally, $\Upsilon_{J-J}^{+(s, t)}$ and $\Upsilon_{J J}^{-(s, t)}$ satisfy the closed equation $\partial \Upsilon+\frac{(2 J+3) s}{c} \Upsilon=0$. Then (A.38) is satisfied and hence they contribute to the index. The eigenvalues of $R$ are $r=2( \pm(2 J+$ $\left.2)+i\left(q_{s i}-q_{t j}\right)\right)$. This gives the second line of (A.33).

\footnotetext{
${ }^{18}$ In fact, these modes are the complex conjugate of the highest modes with $m=J$.
} 


\section{B The saddle point equation}

In this appendix, we derive (4.10). We start with the effective action of (4.7),

$$
\begin{aligned}
S_{\mathrm{eff}}= & \beta\left(1-\int_{-q_{m}}^{q_{m}} d q \rho(q)\right)+\frac{2 N_{5}}{\lambda} \int_{-q_{m}}^{q_{m}} d q q^{2} \rho(q) \\
& -\frac{1}{2} \int_{-q_{m}}^{q_{m}} d q \int_{-q_{m}}^{q_{m}} d q^{\prime} \rho(q) \rho\left(q^{\prime}\right) \sum_{J=0}^{N_{5}-1} \log \frac{\left\{(2 J+2)^{2}+\left(q-q^{\prime}\right)^{2}\right\}\left\{(2 J)^{2}+\left(q-q^{\prime}\right)^{2}\right\}}{\left\{(2 J+1)^{2}+\left(q-q^{\prime}\right)^{2}\right\}^{2}} .
\end{aligned}
$$

Here, $\lambda=g^{2} N_{2}$ is the 't Hooft coupling and $\beta$ is the Lagrange multiplier for the normalization of the eigenvalue density (4.9). The saddle point equation is obtained by differentiating $S_{\text {eff }}$ with respect to $\rho(q)$ and is given by

$$
\beta=\frac{2 N_{5}}{\lambda} q^{2}-\int_{-q_{m}}^{q_{m}} d q^{\prime} \rho\left(q^{\prime}\right) \sum_{J=0}^{N_{5}-1} \log \frac{\left\{(2 J+2)^{2}+\left(q-q^{\prime}\right)^{2}\right\}\left\{(2 J)^{2}+\left(q-q^{\prime}\right)^{2}\right\}}{\left\{(2 J+1)^{2}+\left(q-q^{\prime}\right)^{2}\right\}^{2}} .
$$

Here the integral of $q^{\prime}$ should be understood as the principal value.

We first consider the following identity,

$$
\log \tanh ^{2}\left(\frac{\pi x}{2}\right)=\log \left(\frac{\pi x}{2}\right)^{2}+\sum_{J=1}^{\infty}\left(1+\frac{x^{2}}{(2 J)^{2}}\right)^{2}-\sum_{J=1}^{\infty}\left(1+\frac{x^{2}}{(2 J-1)^{2}}\right)^{2},
$$

which follows from the infinite product expression of the hyperbolic sine and cosine functions. By using this identity, we find that the second term in (B.2) can be written as

$$
-\int_{-q_{m}}^{q_{m}} d q^{\prime} \rho\left(q^{\prime}\right)\left[\log \tanh ^{2}\left(\frac{\pi\left(q-q^{\prime}\right)}{2}\right)-\sum_{J=N_{5}}^{\infty} \log \frac{\left\{(2 J+2)^{2}+\left(q-q^{\prime}\right)^{2}\right\}\left\{(2 J)^{2}+\left(q-q^{\prime}\right)^{2}\right\}}{\left\{(2 J+1)^{2}+\left(q-q^{\prime}\right)^{2}\right\}^{2}}\right]
$$

up to a constant term. We ignore the constant term since it can always be absorbed by a redefinition of $\beta$. In the regime where $N_{5}$ is finite but $\lambda$ is very large, $q_{m}$ also becomes very large. To see the $q_{m}$-dependence clearly, let us rescale the variables as $q=q_{m} \xi$. From the fact that

$$
q_{m} \log \tanh ^{2} \frac{\pi q_{m} \xi}{2} \rightarrow-\pi \delta(\xi) \quad\left(q_{m} \rightarrow \infty\right),
$$

we find that the first term in (B.4) is equal to $\pi \rho(q)$ in this limit. In the second term in (B.4), we approximate the discrete sum with a continuous integral by replacing $J / q_{m} \rightarrow \eta$ and $\sum_{J=N_{5}}^{\infty} \rightarrow q_{m} \int_{N_{5} / q_{m}}^{\infty} d \eta$. Then, the second term can be evaluated as

$$
\begin{aligned}
& -2 \int_{-1}^{1} d \xi^{\prime} \rho\left(q_{m} \xi^{\prime}\right) \int_{N_{5} / q_{m}}^{\infty} d \eta\left[\frac{4 \eta^{2}-\left(\xi-\xi^{\prime}\right)^{2}}{\left(4 \eta^{2}+\left(\xi-\xi^{\prime}\right)^{2}\right)^{2}}+\mathcal{O}\left(1 / q_{m}\right)\right] \\
& \simeq-\int_{-q_{m}}^{q_{m}} d q^{\prime} \rho\left(q^{\prime}\right) \frac{2 N_{5}}{\left(2 N_{5}\right)^{2}+\left(q-q^{\prime}\right)^{2}} .
\end{aligned}
$$

Thus, in the strongly coupled regime, the saddle point equation (B.2) is reduced to (4.10). 


\section{Solving the saddle point equation}

In this appendix, we construct solutions of the saddle point equations of the eigenvalue integrals obtained by the localization.

\section{C.1 For the simplest partition}

Here, we derive (4.11). We first rewrite (4.10) into a more tractable form. We define the resolvent by

$$
\omega(z)=\int_{-q_{m}}^{q_{m}} d q \frac{\rho(q)}{z-q} .
$$

For $q \in\left[-q_{m}, q_{m}\right]$, this satisfies

$$
\omega(q \pm i 0)=P \int_{-q_{m}}^{q_{m}} d q^{\prime} \frac{\rho\left(q^{\prime}\right)}{q-q^{\prime}} \mp \pi i \rho(q),
$$

where $P \int_{-q_{m}}^{q_{m}}$ denotes the principal value. Note that the last term in (4.10) can be written as $\frac{1}{2 i}\left\{\omega\left(q-2 i N_{5}\right)-\omega\left(q+2 i N_{5}\right)\right\}$. By using this and (C.2), we rewrite (4.10) as

$$
\beta=\frac{1}{2 i}\left\{\omega\left(q+2 i N_{5}\right)-\omega(q+i 0)\right\}-\frac{1}{2 i}\left\{\omega\left(q-2 i N_{5}\right)-\omega(q-i 0)\right\}+\frac{2 N_{5}}{\lambda} q^{2} .
$$

When $q_{m}$ is large compared to $N_{5}$, we can expand $\omega\left(q \pm 2 i N_{5}\right)$ as

$$
\omega\left(q \pm 2 i N_{5}\right)=\omega(q \pm i 0) \pm 2 i N_{5} \omega^{\prime}(q \pm i 0)+\cdots .
$$

The convergence of this expansion can be seen clearly if one rescales the variable as $q=q_{m} \xi$, as we did in appendix B. Thus, in the large- $q_{m}$ limit, the equation (C.3) becomes

$$
\beta=N_{5}\left\{\omega^{\prime}(q+i 0)+\omega^{\prime}(q-i 0)\right\}+\frac{2 N_{5}}{\lambda} q^{2} .
$$

By integrating this equation, we obtain

$$
\omega(q+i 0)+\omega(q-i 0)=\frac{\beta}{N_{5}} q-\frac{2}{3 \lambda} q^{3},
$$

where we have set the integration constant to be zero because of the symmetry under $q \rightarrow-q$.

The equation (C.6) is identical with the equation of motion of the quartic one matrix model. Hence, the solution takes the same form as the quartic matrix model, where the resolvent is written as

$$
\omega(z)=\frac{1}{2}\{\omega(q+i 0)+\omega(q-i 0)\}+\left(a+b z^{2}\right) \sqrt{z^{2}-q_{m}^{2}} .
$$

We substitute (C.6) into this expression. Then, the asymptotic behavior of the resolvent, $\omega(z) \rightarrow \frac{1}{z}(z \rightarrow \infty)$, gives three conditions, which enable us to express $a, b$ and $\beta$ in terms of $q_{m}$ :

$$
a=-\frac{2}{q_{m}^{2}}+\frac{2 q_{m}^{2}}{3 \lambda}, \quad b=\frac{1}{3 \lambda}, \quad \frac{\beta}{N_{5}}=\frac{4}{q_{m}^{2}}+\frac{q_{m}^{2}}{2 \lambda} .
$$


Thus, the resolvent is finally determined as

$$
\omega(z)=\left(\frac{2}{q_{m}^{2}}+\frac{q_{m}^{2}}{4 \lambda}\right) z-\frac{1}{3 \lambda} z^{3}-\left(\frac{2}{q_{m}^{2}}+\frac{q_{m}^{2}}{12 \lambda}-\frac{z^{2}}{3 \lambda}\right) \sqrt{z^{2}-q_{m}^{2}} .
$$

The eigenvalue density is given by the discontinuity of (C.9) as

$$
\rho(q)=\frac{1}{\pi}\left(\frac{2}{q_{m}^{2}}+\frac{q_{m}^{2}}{12 \lambda}-\frac{q^{2}}{3 \lambda}\right) \sqrt{q_{m}^{2}-q^{2}} .
$$

Note that in order for $\rho(q)$ to be positive for any $q \in\left[-q_{m}, q_{m}\right], q_{m}$ has to satisfy

$$
q_{m}^{4} \leq 8 \lambda
$$

Finally, we determine the value of $q_{m}$ from the action principle. By using the saddle point equation, we can reduce the effective action (B.1) to

$$
S_{\mathrm{eff}} /\left(N_{2}\right)^{2}=\frac{N_{5}}{\lambda} \int_{-q_{m}}^{q_{m}} q^{2} \rho(q)+\frac{\beta}{2}
$$

By evaluating this using (C.10), we obtain the on-shell value of the effective action as

$$
S_{\mathrm{eff}} /\left(N_{2}\right)^{2}=\frac{2 N_{5}}{q_{m}^{2}}\left(1+\frac{q_{m}^{4}}{4 \lambda}-\frac{q_{m}^{8}}{192 \lambda^{2}}\right) .
$$

In the region (C.11), the minimum of $S_{\text {eff }}$ is realized at

$$
q_{m}^{4}=8 \lambda
$$

By substitute this into (C.10), we obtain (4.11).

\section{C.2 For the generic partition}

Here, we construct a solution to (4.18) in the decoupling limit of M5-branes.

In the decoupling limit, by applying the same computation that we used to derive (C.5), we can reduce the saddle point equations (4.18) to

$$
\frac{1}{2} \sum_{t=1}^{\Lambda}\left(n_{s}+n_{t}-\left|n_{s}-n_{t}\right|\right)\left(\omega_{t}^{\prime}(q+i 0)+\omega_{t}^{\prime}(q-i 0)\right)=\beta_{s}-\frac{2 n_{s}}{g^{2}} q^{2},
$$

where we have defined the resolvent as

$$
\omega_{s}(z)=\int_{-q_{s}}^{q_{s}} d q \frac{\rho_{s}(q)}{z-q} .
$$

Without loss of generality, we assume that $n_{s}$ in the decomposition (1.2) are ordered as $n_{1}>n_{2}>\cdots>n_{\Lambda}$. We also assume that

$$
q_{\Lambda}>q_{\Lambda-1}>\cdots>q_{1} .
$$


Then, let us first consider the equation (C.15) with $s=\Lambda$,

$$
n_{\Lambda} \sum_{t=1}^{\Lambda}\left(\omega_{t}^{\prime}(q+i 0)+\omega_{t}^{\prime}(q-i 0)\right)=\beta_{\Lambda}-\frac{2 n_{\Lambda}}{g^{2}} q^{2}, \quad q \in\left[-q_{\Lambda}, q_{\Lambda}\right] .
$$

Under the assumption (C.17), it makes sense to consider

$$
\hat{\rho}_{\Lambda}(q)=\sum_{s=1}^{\Lambda} \rho_{s}(q)
$$

which has the support $\left[-q_{\Lambda}, q_{\Lambda}\right]$ and is normalized as

$$
\int_{-q_{\Lambda}}^{q_{\Lambda}} d q \hat{\rho}_{\Lambda}(q)=\sum_{s=1}^{\Lambda} N_{2}^{(s)}
$$

In terms of $\hat{\rho}_{\Lambda}(q),(\mathrm{C} .18)$ can be simply written as

$$
n_{\Lambda}\left(\hat{\omega}_{\Lambda}^{\prime}(q+i 0)+\hat{\omega}_{\Lambda}^{\prime}(q-i 0)\right)=\beta_{\Lambda}-\frac{2 n_{\Lambda}}{g^{2}} q^{2}, \quad q \in\left[-q_{\Lambda}, q_{\Lambda}\right],
$$

where $\hat{\omega}_{\Lambda}(z)$ is the resolvent for $\hat{\rho}_{\Lambda}(q)$. Since (C.21) takes the same form as (C.5), the solution for $\rho_{\Lambda}(q)$ is also given by the same form as (C.10). We will determine $q_{\Lambda}$ below.

Next, we solve (C.15) with $s<\Lambda$. Let us consider the difference between (C.15) with $s=r$ and (C.15) with $s=r+1$ on the support $q \in\left[-q_{r}, q_{r}\right]$, where $r \in\{1,2, \cdots, \Lambda-1\}$. This leads to

$$
\left(n_{r}-n_{r+1}\right) \sum_{t=1}^{r}\left(\omega_{t}^{\prime}(q+i 0)+\omega_{t}^{\prime}(q-i 0)\right)=\beta_{r}-\beta_{r+1}-\frac{2\left(n_{r}-n_{r+1}\right)}{g^{2}} q^{2}, \quad q \in\left[-q_{r}, q_{r}\right] .
$$

Note that $\rho_{s}(q)$ with $s>r$ does not appear in this equation. We introduce new variables

$$
\hat{\rho}_{r}(q)=\sum_{s=1}^{r} \rho_{s}(q)
$$

which are normalized as

$$
\int_{-q_{r}}^{q_{r}} d q \hat{\rho}_{r}(q)=\sum_{s=1}^{r} N_{2}^{(s)}
$$

In terms of $\hat{\rho}_{r}(q),(\mathrm{C} .22)$ becomes

$$
\left(n_{r}-n_{r+1}\right)\left(\hat{\omega}_{r}^{\prime}(q+i 0)+\hat{\omega}_{r}^{\prime}(q-i 0)\right)=\beta_{r}-\beta_{r+1}-\frac{2\left(n_{r}-n_{r+1}\right)}{g^{2}} q^{2}, \quad q \in\left[-q_{r}, q_{r}\right] .
$$

Again, this is the same form as (C.5), so that the solution for $\hat{\rho}_{r}$ is given by the same form as (C.10).

Finally we determine $q_{r}$. By using the equation of motion for $\rho_{s}$, the on-shell can be computed as

$$
S_{\mathrm{eff}}=\sum_{s=1}^{\Lambda} \frac{2\left(n_{s}-n_{s+1}\right)}{q_{s}^{2}}\left(1+\frac{q_{s}^{4}}{4 \lambda_{s}}-\frac{q_{s}^{8}}{192 \lambda_{s}^{2}}\right)
$$

where $\lambda_{s}=g^{2} \sum_{r=1}^{s} N_{2}^{(r)}$. Thus, the minimum is given by $q_{s}=\left(8 \lambda_{s}\right)^{1 / 4}$. Thus, we obtained (4.19). 


\section{Eigenvalue distribution in the M2-brane limit}

In this appendix, we solve the eigenvalue integral (5.1) for large $N_{2}$. Putting $a=g^{2} N_{2} / N_{5}$, we consider the scaling limit such that $N_{5}, N_{2}, a \rightarrow \infty, N_{5} / N_{2} \rightarrow \infty$ and $N_{2} / a \rightarrow \infty$. We again assume that the typical value of eigenvalues is very large in this limit, since the Gaussian attractive force becomes weak.

We introduce the eigenvalue density $\rho(q)$ as (4.8). The effective action of (5.1) is written in terms of $\rho(q)$ as

$$
\begin{aligned}
S_{\text {eff }} /\left(N_{2}\right)^{2}= & \frac{2 N_{5}}{\lambda} \int_{-q_{m}}^{q_{m}} d q q^{2} \rho(q)-\frac{1}{2} \int_{-q_{m}}^{q_{m}} d q \int_{-q_{m}}^{q_{m}} d q^{\prime} \rho(q) \rho\left(q^{\prime}\right) \log \tanh ^{2}\left(\frac{\pi\left(q-q^{\prime}\right)}{2}\right) \\
& +\beta\left(1-\int_{-q_{m}}^{q_{m}} d q \rho(q)\right) .
\end{aligned}
$$

By applying (B.5), we find that the action reduces to

$$
S_{\mathrm{eff}} /\left(N_{2}\right)^{2}=\frac{2 N_{5}}{\lambda} \int_{-q_{m}}^{q_{m}} d q q^{2} \rho(q)+\frac{\pi}{2} \int_{-q_{m}}^{q_{m}} d q \rho(q)^{2}+\beta\left(1-\int_{-q_{m}}^{q_{m}} d q \rho(q)\right) .
$$

The saddle point equation is given by

$$
\beta=\pi \rho(q)+\frac{2 N_{5}}{\lambda} q^{2}
$$

Thus, $\rho(q)$ is a quadratic function in $q$ and $q_{m}$ is related to $\beta$ as

$$
q_{m}^{2}=\frac{\lambda}{2 N_{5}} \beta
$$

From (4.9), (D.3) and (D.4), we obtain

$$
q_{m}=\left(\frac{3 \pi \lambda}{8 N_{5}}\right)^{\frac{1}{3}}
$$

Thus, the typical value of the eigenvalues should be proportional to $\left(\frac{\lambda}{N_{5}}\right)^{1 / 3}$. Note that this result is consistent with our assumption that the typical value of the eigenvalues is very large in the strong coupling region.

Open Access. This article is distributed under the terms of the Creative Commons Attribution License (CC-BY 4.0), which permits any use, distribution and reproduction in any medium, provided the original author(s) and source are credited.

\section{References}

[1] T. Banks, W. Fischler, S.H. Shenker and L. Susskind, $M$ theory as a matrix model: a conjecture, Phys. Rev. D 55 (1997) 5112 [hep-th/9610043] [INSPIRE].

[2] D.E. Berenstein, J.M. Maldacena and H.S. Nastase, Strings in flat space and pp waves from $N=4$ super Yang-Mills, JHEP 04 (2002) 013 [hep-th/0202021] [INSPIRE]. 
[3] J.M. Maldacena, M.M. Sheikh-Jabbari and M. Van Raamsdonk, Transverse five-branes in matrix theory, JHEP 01 (2003) 038 [hep-th/0211139] [INSPIRE].

[4] J. Madore, The fuzzy sphere, Class. Quant. Grav. 9 (1992) 69 [INSPIRE].

[5] Y. Lozano and D. Rodriguez-Gomez, Fuzzy 5-spheres and pp-wave matrix actions, JHEP 08 (2005) 044 [hep-th/0505073] [INSPIRE].

[6] Y. Asano, G. Ishiki, S. Shimasaki and S. Terashima, Spherical transverse M5-branes in matrix theory, Phys. Rev. D 96 (2017) 126003 [arXiv:1701.07140] [INSPIRE].

[7] W. Taylor, M(atrix) theory: matrix quantum mechanics as a fundamental theory, Rev. Mod. Phys. 73 (2001) 419 [hep-th/0101126] [INSPIRE].

[8] H. Ling, A.R. Mohazab, H.-H. Shieh, G. van Anders and M. Van Raamsdonk, Little string theory from a double-scaled matrix model, JHEP 10 (2006) 018 [hep-th/0606014] [INSPIRE].

[9] K. Dasgupta, M.M. Sheikh-Jabbari and M. Van Raamsdonk, Matrix perturbation theory for M-theory on a PP wave, JHEP 05 (2002) 056 [hep-th/0205185] [INSPIRE].

[10] N. Kim and J. Plefka, On the spectrum of PP wave matrix theory, Nucl. Phys. B 643 (2002) 31 [hep-th/0207034] [INSPIRE].

[11] K. Dasgupta, M.M. Sheikh-Jabbari and M. Van Raamsdonk, Protected multiplets of M-theory on a plane wave, JHEP 09 (2002) 021 [hep-th/0207050] [INSPIRE].

[12] B. de Wit, J. Hoppe and H. Nicolai, On the quantum mechanics of supermembranes, Nucl. Phys. B 305 (1988) 545 [INSPIRE].

[13] M.M. Sheikh-Jabbari and J. Simon, On half-BPS states of the ABJM theory, JHEP 08 (2009) 073 [arXiv:0904.4605] [INSPIRE].

[14] V. Pestun, Localization of gauge theory on a four-sphere and supersymmetric Wilson loops, Commun. Math. Phys. 313 (2012) 71 [arXiv:0712.2824] [INSPIRE].

[15] Y. Asano, G. Ishiki, T. Okada and S. Shimasaki, Exact results for perturbative partition functions of theories with $\mathrm{SU}(2 \mid 4)$ symmetry, JHEP 02 (2013) 148 [arXiv:1211.0364] [INSPIRE].

[16] Y. Asano, G. Ishiki, T. Okada and S. Shimasaki, Emergent bubbling geometries in the plane wave matrix model, JHEP 05 (2014) 075 [arXiv: 1401.5079] [INSPIRE].

[17] Y. Asano, G. Ishiki and S. Shimasaki, Emergent bubbling geometries in gauge theories with SU(2|4) symmetry, JHEP 09 (2014) 137 [arXiv:1406.1337] [INSPIRE].

[18] J.-T. Yee and P. Yi, Instantons of M(atrix) theory in PP wave background, JHEP 02 (2003) 040 [hep-th/0301120] [INSPIRE].

[19] H. Lin, Instantons, supersymmetric vacua and emergent geometries, Phys. Rev. D 74 (2006) 125013 [hep-th/0609186] [INSPIRE].

[20] C. Bachas, J. Hoppe and B. Pioline, Nahm equations, $N=1 *$ domain walls and $D$ strings in $A d S_{5} \times S^{5}, J H E P 07$ (2001) 041 [hep-th/0007067] [INSPIRE].

[21] S. Kovacs, Y. Sato and H. Shimada, On membrane interactions and a three-dimensional analog of Riemann surfaces, JHEP 02 (2016) 050 [arXiv: 1508.03367] [INSPIRE].

[22] J. Polchinski, M theory and the light cone, Prog. Theor. Phys. Suppl. 134 (1999) 158 [hep-th/9903165] [INSPIRE]. 
[23] H. Lin and J.M. Maldacena, Fivebranes from gauge theory, Phys. Rev. D 74 (2006) 084014 [hep-th/0509235] [INSPIRE].

[24] D.E. Berenstein, M. Hanada and S.A. Hartnoll, Multi-matrix models and emergent geometry, JHEP 02 (2009) 010 [arXiv: 0805.4658] [INSPIRE].

[25] V.G. Filev and D. O'Connor, Multi-matrix models at general coupling, J. Phys. A 46 (2013) 475403 [arXiv: 1304 .7723] [INSPIRE].

[26] V.G. Filev and D. O'Connor, On the phase structure of commuting matrix models, JHEP 08 (2014) 003 [arXiv:1402.2476] [InSPIRE].

[27] D. Berenstein, Large-N BPS states and emergent quantum gravity, JHEP 01 (2006) 125 [hep-th/0507203] [INSPIRE].

[28] D. Berenstein, D.H. Correa and S.E. Vazquez, All loop BMN state energies from matrices, JHEP 02 (2006) 048 [hep-th/0509015] [INSPIRE].

[29] G. Ishiki, S. Shimasaki, Y. Takayama and A. Tsuchiya, Embedding of theories with $\mathrm{SU}(2 \mid 4)$ symmetry into the plane wave matrix model, JHEP 11 (2006) 089 [hep-th/0610038] [INSPIRE].

[30] T. Ishii, G. Ishiki, S. Shimasaki and A. Tsuchiya, Fiber bundles and matrix models, Phys. Rev. D 77 (2008) 126015 [arXiv: 0802.2782] [INSPIRE].

[31] T. Ishii, G. Ishiki, S. Shimasaki and A. Tsuchiya, $N=4$ super Yang-Mills from the plane wave matrix model, Phys. Rev. D 78 (2008) 106001 [arXiv:0807.2352] [INSPIRE]. 